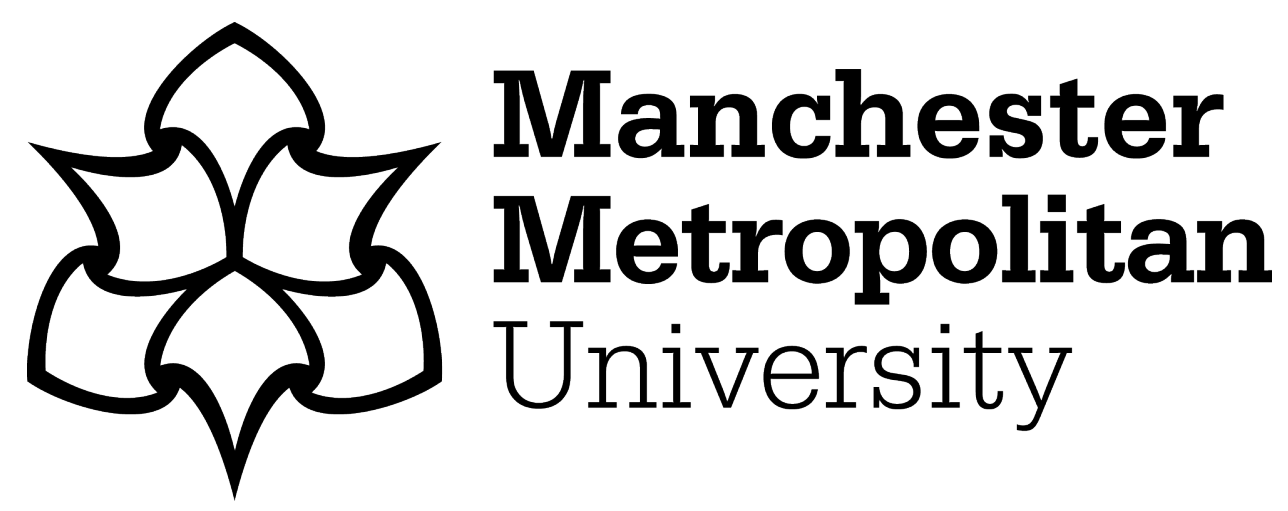

Martin, Sarah L ORCID logoORCID: https://orcid.org/0000-0001-6382-3977, Jones, Anthony KP, Brown, Christopher A, Kobylecki, Christopher and Silverdale, Monty A (2019) A neurophysiological investigation of anticipation to pain in Parkinson's disease. European Journal of Neuroscience, 51 (2). pp. 611-627. ISSN 0953-816X

Downloaded from: https://e-space.mmu.ac.uk/625272/

Version: Accepted Version

Publisher: Wiley

DOI: https://doi.org/10.1111/ejn.14559

Please cite the published version 
Research Report

Running Author: SARAH et al.

\title{
A neurophysiological investigation of anticipation to pain in Parkinson's disease
}

\author{
Martin L. Sarah ${ }^{1}$ \\ Jones K. P. Anthony ${ }^{1}$ \\ Brown A. Christopher 2 \\ Kobylecki Christopher ${ }^{3}$ \\ Silverdale A. Monty ${ }^{3}$ \\ AQ1
}

1 The Human Pain Research Group, Division of Neuroscience and Experimental Psychology, University of Manchester, Manchester, UK

2 Department of Psychological Sciences, University of Liverpool, Liverpool, UK

3 Institution is Department of Neurology, Salford Royal NHS Foundation Trust, Manchester Academic Health Science Centre, The University of Manchester, Manchester, UK AQ2

\section{Correspondence}

Sarah Martin, The Human Pain Research Group, Salford Royal NHS Foundation Trust Hospital, The University of Manchester, Room B204, Manchester, UK. Email: sarah.martin-10@postgrad.manchester.ac.uk

\begin{abstract}
Chronic pain is common in people with Parkinson's disease and is often considered to be caused by the motor impairments associated with the disease. Altered top-down processing of pain characterises several chronic pain conditions and occurs when the cortex modifies nociceptive processing in the brain and spinal cord. This contrasts with bottom-up modulation of pain whereby nociceptive processing is modified on its way up to the brain. Although several studies have demonstrated altered bottom-up pain processing in Parkinson's, the contribution of enhanced anticipation to pain and atypical top-down processing of pain has not been fully explored. During the anticipation to noxious stimuli, EEG source localisation reported an increased activation in the midcingulate cortex and supplementary motor area in the Parkinson's disease group compared to the healthy control group during
\end{abstract}


mid [-1,500 -1,000]-and late anticipation [-500 0], indicating enhanced cortical activity before noxious stimulation. The Parkinson's disease group was also more sensitive to the laser and required a lower voltage level to induce pain. This study provides evidence supporting the hypothesis that enhanced top-down processing of pain may contribute to the development of chronic pain in Parkinson's. Additional research to establish whether the altered anticipatory response is unique to noxious stimuli is required as no control stimulus was used within the current study. With further research to confirm these findings, our results inform a scientific rationale for novel treatment strategies of pain in Parkinson's disease, including mindfulness, cognitive therapies and other approaches targeted at improving top-down processing of pain.

\section{Graphical Abstract}

Parkinson's patients showed an increased activation in the midcingulate and supplementary motor area during the anticipation of noxious stimuli. The augmented anticipation was independent of motor impairment, chronic pain severity, mood and catastrophising behaviours. Therefore, demonstrating the high prevalence of chronic pain in Parkinson's may be caused by altered central pain processing.

\section{(a) Mid-anticipation [-1,500-1,000 ms]}

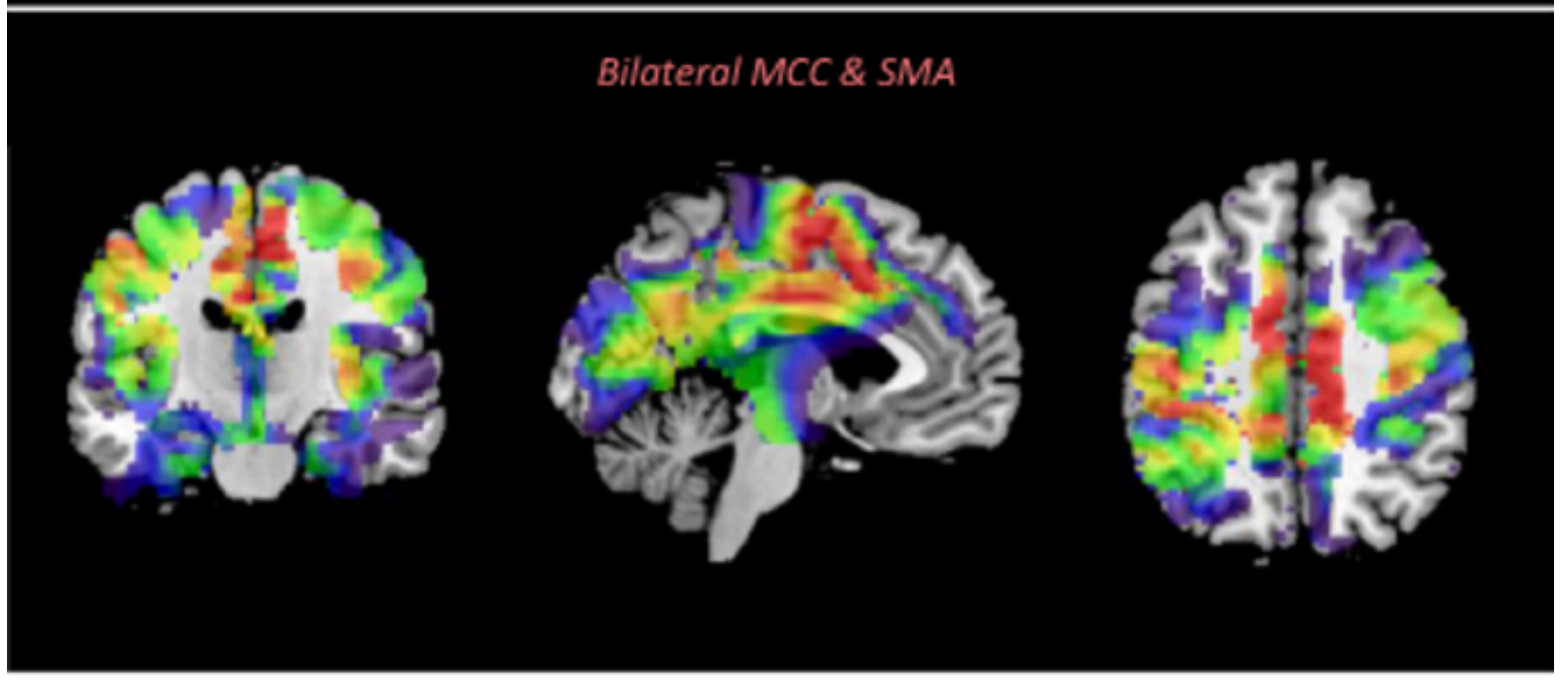

(b) Late anticipation $[-5000 \mathrm{~ms}]$ 

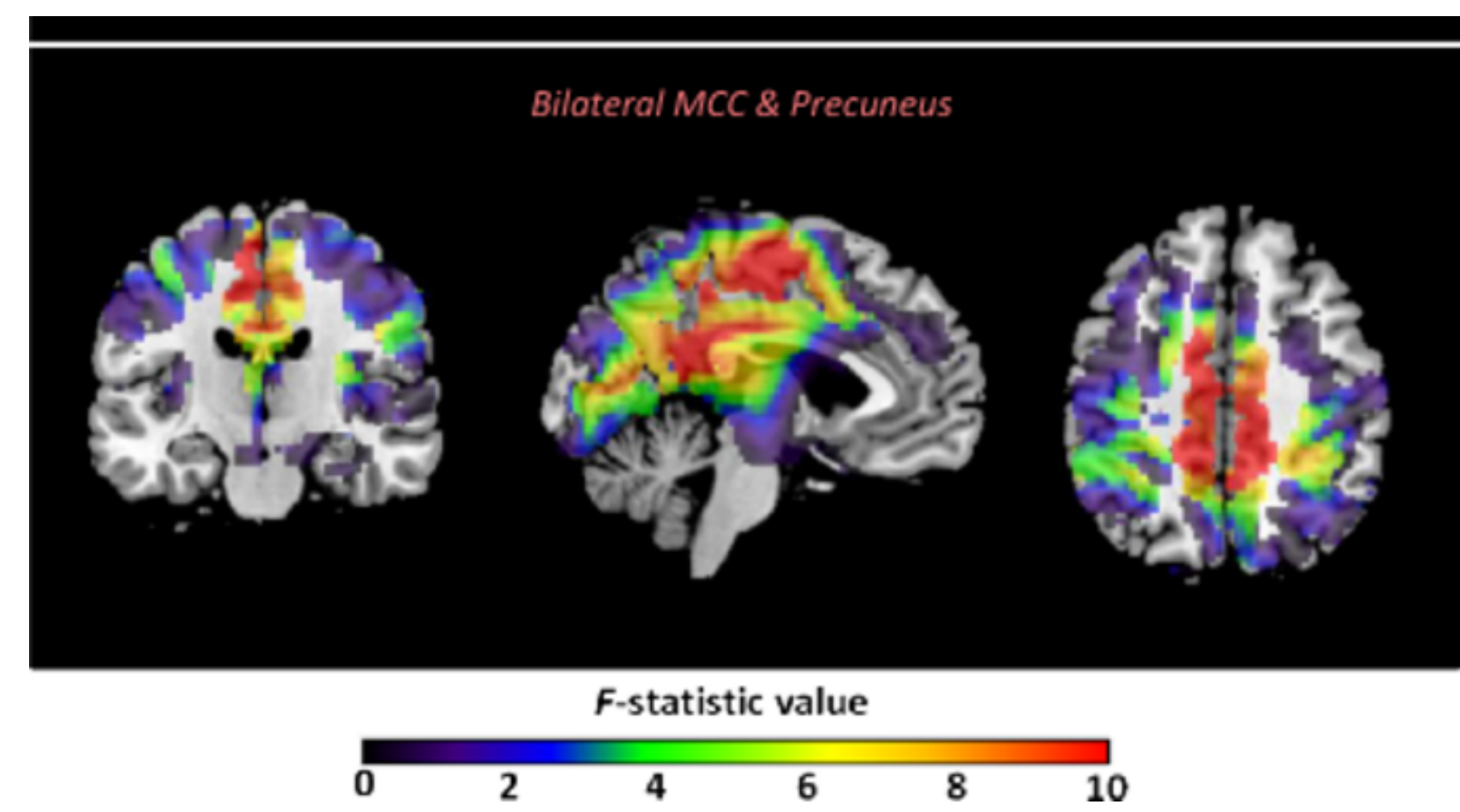

Received: 02 February 2019 | Revised: 23 July 2019 | Accepted: 15 August 2019

\section{Keywords}

brain; EEG; laser; nociception; source localisation

Edited by Guillaume Rousselet. Reviewed by Xxxxxx.

Funding informationSM was funded by Parkinson's UK and the research carried out in The Human Pain Research Group, The University of Manchester.

\section{Abbreviations}

AAL2

BEM

$\mathrm{BL}$ anatomical automatic labelling boundary element model baseline 


$\begin{array}{ll}\text { CO2 } & \text { carbon dioxide } \\ \text { EEG } & \text { electroencephalogram } \\ \text { fMRI } & \text { functional magnetic resonance imaging } \\ \text { HADS } & \text { hospital anxiety and depression scale } \\ \text { HC } & \text { healthy control } \\ \text { LEP } & \text { laser-evoked potential } \\ \text { LORETA } & \text { low resolution electromagnetic tomography } \\ \text { MCC } & \text { midcingulate cortex } \\ \text { MDS-UPDRS } & \text { Movement Disorder Society Unified Parkinson's Disease Rating Scale } \\ \text { MoCA } & \text { montreal cognitive assessment } \\ \text { PAG } & \text { periaqueductal grey } \\ \text { PCS } & \text { pain catastrophising scale } \\ \text { PD } & \text { Parkinson's disease } \\ \text { PET } & \text { positron emission topography } \\ \text { PFC } & \text { prefrontal cortex } \\ \text { POI } & \text { period of interest } \\ \text { PWPD } & \text { people with Parkinson's disease } \\ \text { SASICA } & \text { semiautomatic selection of independent components for artifact correction } \\ \text { SD } & \text { standard deviation } \\ \text { SI/SII } & \text { somatosensory cortex } \\ \text { SMA } & \text { supplementary motor area } \\ \text { SPM } & \text { statistical parametric mapping } \\ \text { TWOI } & \text { time window of interest } \\ \text { VAS } & \text { visual analogue scale } \\ \end{array}$

\section{INTRODUCTION}

\subsection{Pain in Parkinson's disease}

Chronic pain is a highly prominent symptom in people with Parkinson's disease (PwPD), yet there is a limited understanding of whether the pain is primarily a consequence of motor impairment, including muscle rigidity, or whether Parkinson's disease (PD) causes a centrally produced heightened sensitivity to pain. Whilst the percentage of the general population living with chronic pain is approximately 20\% (Breivik, Collett, Ventafridda, Cohen, \& Gallacher, 2006; van Hecke, Torrance, \& Smith, 2013), there is a significantly 
higher prevalence within the PD population of approximately two-thirds (Nègre-Pagès, Regragui, Bouhassira, Grandjean, \& Rascol, 2008; Ozturk, Gundogdu, Kocer, Comoglu, \& Cakci, 2016; Silverdale et al., 2018; Skogar \& Lokk, 2016). There is evidence that PwPD have lower threshold and tolerance of pain compared to age-matched healthy cohorts (Brefel-Courbon et al., 2005; Chaudhuri \& Schapira, 2009; Djaldetti et al., 2004; Schestatsky et al., 2007), and EEG and functional imaging studies have demonstrated an altered central response to pain in PD (Brefel-Courbon et al., 2005; Schestatsky et al., 2007; Tinazzi et al., 2009). These abnormalities provide strong evidence that altered central pain processing contributes to the development of pain in PD (Silverdale et al., 2018). Therefore, an increased understanding of the pathophysiological mechanisms causing the chronic pain is imperative to make an informed improvement in the treatment of pain in Parkinson's.

\subsection{Top-down alteration in pain processing}

Research into chronic pain has largely focused on bottom-up mechanisms amplifying the nociceptive information on its way from the peripheries to the brain (Reicherts et al., 2017; Watson et al., 2009). Bottom-up processing refers to the transmission and encoding of sensory information from noxious stimuli, which is not affected by subjective modulation of pain perception. However, there is a developing field in the role of top-down modulation of pain, whereby the cortical activity modulates the nociceptive information.

Previous research within our group has used EEG source localisation to investigate the anticipatory processing of painful stimuli. Our previous research has shown that the anticipation of pain leads to changes in the activity of the same brain regions that subsequently respond to nociception (Brown, El-Deredy, \& Jones, 2014; Brown \& Jones, 2008; Brown, Seymour, Boyle, El-Deredy, \& Jones, 2008; Clark, Brown, Jones, \& El-Deredy, 2008). Examples of regions which have been seen to activate during anticipation include the cingulate cortex, insula, primary and secondary somatosensory cortex (SI/SII), prefrontal cortex (PFC) and the periaqueductal grey (PAG) (Babiloni et al., 2004; Koyama, McHaffie, Laurienti, \& Coghill, 2005). The degree of anticipation has been shown to be correlated with subsequent pain perception and the EEG laser-evoked potential (LEP) (Brown, Seymour, Boyle, et al., 2008). Hence, we have previously demonstrated that the cortex can modulate the perception of pain of the incoming pain information via top-down modulation. Within chronic pain conditions, such as fibromyalgia and osteoarthritis, heightened anticipation within the insula cortex is correlated with the extent and severity of chronic pain symptoms (Brown et al., 2014).

Unlike fMRI and PET, the higher temporal resolution of EEG allows for a more accurate recording of the anticipatory processes. Prior to a noxious stimulus, during anticipation, there is cortical activity from a network of regions including the cingulate cortex, basal ganglia and thalamic structures (Brunia \& van Boxtel, 2001; Vogt, Finch, \& Olson, 1992). In this study, we used EEG with source localisation analysis because of the temporal advantage over neuroimaging techniques reliant on slow haemodynamic responses. The accuracy and reliability of EEG source localisation has been verified by research which shows high similarity to other neuroimaging techniques such as PET (Lantz, Grave de Peralta, Spinelli, Seeck, \& Michel, 2003; Michel et al., 2004), fMRI (Mulert et al., 2004; Vitacco, Brandeis, PascualMarqui, \& Martin, 2002) and intracerebral recordings (Seeck et al., 1998; Trébuchon-Da Fonseca, Giraud, Badier, Chauvel, \& LiégeoisChauvel, 2005). EEG source localisation has reliably reported activations within the regions associated with pain perception during anticipation (Brown \& Jones, 2012; Brown, Seymour, El-Deredy, et al., 2008; Brown, Seymour, Boyle, et al., 2008). For instance, impaired processing within the parietal and frontal regions during anticipation has been reported using source localisation in patients with the chronic pain associated with fibromyalgia and osteoarthritis (Brown et al., 2014). 


\subsection{Aim of study}

Here, we investigated anticipatory processing in PWPD in the 'off' medication state in comparison to age-matched healthy volunteers. We used a $\mathrm{CO}_{2}$ laser to induce acute noxious stimuli and monitored brain activity throughout via EEG. We hypothesised that the anticipatory phase would be abnormal in the PD group and would provide evidence that top-down mechanisms are key to explaining the mechanisms of chronic pain in PD.

\section{METHODS}

The study was approved by the local ethics committee, and all participants gave informed consent according to the Declaration of Helsinki to participate in the study. The study was registered with the National Institute of Health Research (NIHR) Central Portfolio Management System (CPMS) (CPMS ID: 19963).

\subsection{Participants}

Twenty-four participants with Parkinson's were recruited (sixteen males). PD patients were recruited via correspondence with their neurologist (MS or CK). Participants were screened to ensure safe withdrawal of medication for the study, and to exclude cases of severe tremors which might interfere with EEG recording. Clinically significant peripheral neuropathy was excluded by clinical examination and neuropathy scale. Symptom duration ranged from 1 month to 16 years, with a mean duration of 5.13 years. One PD participant was unable to complete the study due to severe symptoms after medication withdrawal. Twenty-three participants with PD were included for the analysis of behavioural measures. Twenty PD participants were included for EEG analysis due to two data sets being removed because of noisy data and one for a low MoCA (Montreal Cognitive Assessment) score (12/30).

Twenty-five age-matched healthy controls (HCs) were recruited (fourteen males). One HC participant was unable to complete the study due to the laser failing to induce a sufficient pain level, and one HC data set was removed due to noisy data. Twenty-four HCs were included in the behavioural measures, and $23 \mathrm{HC}$ participants were included for EEG data analysis. The participants with PD [age range: min 46, max 83, median: 66, mean $\pm S D$ : $63.3 \pm 8.27$ years] were age-matched to the HCs [age range: min 45, max 82, median: 63 , mean $\pm S D: 65.5 \pm 8.59$ years].

\subsubsection{Medication}

The PD group omitted their evening medication and were studied in the practically defined OFF state (after $12 \mathrm{hr}$ of withdrawal of antiParkinsonian medication). All analgesic medication was withdrawn $12 \mathrm{hr}$ prior to the study visit. The motor section of the Movement Disorder Society Unified Parkinson's Disease Rating Scale (MDS-UPDRS) was completed to report the current severity of their parkinsonian disability off their medication.

\subsubsection{Assessments}


The participants were assessed for PD motor severity, pain, mood and cognitive state. PD motor severity. The motor section (III) of the MDS-UPDRS was completed to evaluate the motor disability due to PD (Goetz et al., 2008). The assessment in the PD group was completed off their medication. Pain: All participants rated their current pain state via a visual analogue scale (VAS) prior to starting the laser protocol. The PD participants with chronic pain reported their minimum and maximum degree of pain over the last 6 months via a visual analogue scale (VAS). All participants completed the pain catastrophising scale (PCS) (Sullivan et al., 1995) to report their psychological coping ability when experiencing pain. Mood: All participants completed the Hospital Anxiety and Depression Scale (HADS) (Zigmond \& Snaith, 1983) to dissociate results from anxiety or depression. Cognitive state: The Montreal Cognitive Assessment (MoCA) (Nasreddine et al., 2005) test was carried out to assess the participants' cognitive ability. Participants with low scores (<25) were removed from the study to avoid cognitive decline affecting the EEG signal.

AQ3

AQ4

\subsection{Experimental design}

\subsubsection{Pain stimuli}

A $\mathrm{CO}_{2}$ laser [50W Synrad 48-5J-series (J-48-5(S)W) Wavelength: 10,600 nm] was used to deliver acute pain to the dorsal surface of the right forearm. The $\mathrm{CO}_{2}$ laser delivered a beam with a diameter of $15 \mathrm{~mm}$ and $150 \mathrm{~ms}$ duration. The voltage (V) of the laser is linearly related to the laser voltage delivered to the forearm. For each test, the stimuli were delivered in an area measuring $4 \times 5 \mathrm{~cm}$ and were delivered in a pre-determined randomised path (Brown, Seymour, Boyle, et al., 2008). This was to avoid habituation, sensitisation or skin damage.

\subsubsection{Psychophysics}

Before starting the experimental protocol, psychophysics was used to calibrate the laser to the individual's pain sensitivity. An ascending method of limits procedure was used, starting from $0.6 \mathrm{~V}$ with 0.06 increments each time. The participant used an elevenpoint VAS (0-10) to rate the intensity of the pain perceived for each stimuli and the following description was provided; $0=$ no sensation, 4 = pain threshold, 7 = moderately painful, 10 = unbearably painful. The rating scale was introduced to the participant via these standardised descriptives to ensure that no explanation altered their interpretation of the scale. The procedure was repeated three times to allow participants to get used to the laser and was used to calculate the average voltage to induce level 4 (low) and level 7 (moderate) pain. These two levels provided 'low' and 'high' stimuli intensity for the main laser experiment protocol.

\subsubsection{Main experiment}

The participants received 120 laser stimuli at the two intensities (low and high) separated into four conditions; low (level 4), high (level 7), unknown low (level 4) and unknown high (level 7). To investigate the anticipation of a painful stimulus, a 3-s countdown was delivered by three auditory cues at $1 \mathrm{~s}$ intervals (see Figure 1). The first auditory cue was presented concurrently with an anticipatory cue to indicate the forthcoming laser stimuli and to maintain attention. The participant was either shown 'Low', 'High' or 'Unknown'. The presentation of the word 'Unknown' indicated that the laser stimulus has an equal chance of being low or high. This was to investigate 
the importance of certainty in the anticipation of the laser stimuli. The image was also used as a visual fixation cue to discourage eye movements. After the laser stimuli, the 0-10 numerical rating scale was shown on the screen and the participant rated the intensity of the pain. The order of the stimuli was randomised and separated into three blocks with short breaks in-between.

Fig. 1

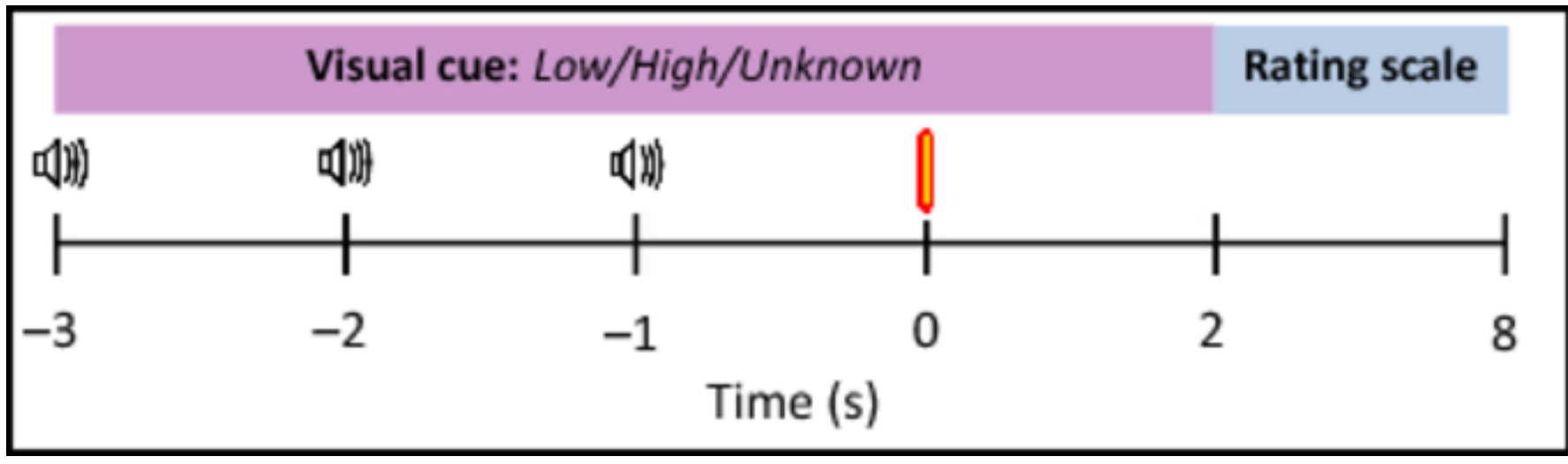

A schematic diagram of a single trial of the experimental paradigm. A computer monitor showed the participant a visual cue of low, high or unknown from $-3 \mathrm{~s}$ to $+2 \mathrm{~s}$. A 3-s countdown of beeps at $-3,-2$ and -1 allowed accurate anticipation of the laser stimuli at time 0 s (red bar). The presentation of the visual cue at $-3 \mathrm{~s}$ was concurrent with the first auditory cue. The visual cue was consistent throughout the anticipation and laser stimulus. At $+2 \mathrm{~s}$, an eleven-point visual analogue scale (VAS) was presented for the participant to rate the laser stimulus. For each condition (low, high, unknown low and unknown high), there were thirty trials. The trials were presented in a randomised order and divided into three blocks of forty trials

\subsubsection{EEG recording}

A BrainVision MR EEG cap was used to record from 63 scalp electrodes using a BrainVision cap system [Standard BrainCap-MR with Multitrodes]. The arrangement of the electrodes was modelled on the extended 10-20 system. Recording parameters were set at filter (DC to $70 \mathrm{~Hz}$ ), sampling rate $(1,000 \mathrm{~Hz})$ and gain (500). To reduce electrical interference, a $50-\mathrm{Hz}$ notch filter was applied. Prior to starting the laser protocol, resting states were recorded with eyes open and closed for $2 \mathrm{~min}$ in all participants. This ensured that the experience prior to the experiment was identical. The resting state data will be analysed and reported separately. The three experimental blocks were recorded separately to allow for better artefact rejection of the EEG data.

\subsection{Analysis methods}

\subsubsection{Statistical analysis of behavioural data}


Statistical analyses of the behavioural measures were carried out using IBM SPSS Statistics 22 software. The questionnaires were all investigated for significant group differences. Prior to using statistical tests, the data were assessed for normality using a combination of Q-Q plots, histograms, and the values of skew and kurtosis. Normally distributed data were analysed using independent $t$ tests and ANOVA tests, whilst data reported to be not normally distributed (namely the PCS, HADS, laser voltage to induce level 7 pain), a nonparametric test was utilised. Specific statistical tests for each analysis step are reported in the results section.

\subsubsection{EEG analysis method $A Q 5$}

EEG pre-processing was carried out using EEGLAB toolbox (Delorme \& Makeig, 2004) in MATLAB version R2015a (the MathWorks Inc), whilst statistical analysis was carried out using SPM12 toolbox (Wellcome Department of Imaging Neuroscience, Institute of Neurology, UCL, London, United Kingdom) running in MATLAB. The EEG data were pre-processed for scalp and source localisation analysis. The main motivation of the analysis was to establish the anatomical origin of the brain activity using (low-resolution electromagnetic tomography) LORETA source localisation.

\section{EEGLAB pre-processing}

Pre-processing consisted of removal and interpolation of bad channels, down-sample to $500 \mathrm{~Hz}$, low-pass filter of $20 \mathrm{~Hz}$ and re-reference to the common average. The four conditions were separated and $-3,500$ ms to 2,000 ms epochs extracted and linear detrend applied. Independent component analysis (ICA) was carried out on all data sets using the SemiAutomatic Selection of Independent Components for Artifact correction (SASICA) toolbox to select components to remove via pre-determined thresholds. The thresholds were set to autocorrelation (threshold $=0.35 \mathrm{r}$, lag $=20 \mathrm{~ms}$ ), focal (threshold $=3.5 \mathrm{z}$ ), focal trial (threshold $5.5 \mathrm{z}$ ), signal to noise (period of interest $(\mathrm{POI})=[0 \mathrm{Inf}]$, baseline $(\mathrm{BL})[-\mathrm{Inf} 0]$, threshold ratio $=0.5)$, and adjust selection enabled. The thresholds were sufficient to remove artefacts from the majority of the data sets; however, a number of data sets required further manual removal of eye-blink components where not picked up by SASICA.

\section{SPM EEG analysis}

The pre-processed data sets were converted to statistical parametric mapping (SPM) compatible files. Statistical analysis was carried out to investigate the anticipation-evoked potentials and the post-stimulus LEPs using scalp-level and source localisation analysis techniques available in the SPM toolbox.

SPM scripts for batch processing were used to analyse the EEG data at the scalp-level and source localisation. Two baselining methods were applied to the data analysis for the anticipation phase and were applied for scalp-level and source localisation analysis. Primary analysis applied distinct baselines (BLs) of $500 \mathrm{~ms}$, occurring prior to each of the three auditory cues, respectively, to analyse each of the three anticipation phases. The BLs and time window of interest (TWOI) were as follows: early [BL: $-3,500 \mathrm{~ms}-3,000 \mathrm{~ms}$ : TWOI: $-2,500 \mathrm{~ms}$ $-2,000 \mathrm{~ms}$ ], mid [BL: $-2,500 \mathrm{~ms}-2,000 \mathrm{~ms}$ : TWOI: $-1,500-1,000 \mathrm{~ms}$-and late [BL: $-1,500-1,000 \mathrm{~ms}$ : TWOI: -500 ms] anticipation phases. The aim of baselining uniquely for each anticipation window was to reduce variability in the data as the anticipation phase progressed, such that the analysis of each phase of anticipation was unique to that phase and not subject to variability arising from 
neural activity occurring in the previous phase. The secondary analysis method applied a single baseline of $500 \mathrm{~ms}$ prior to the first auditory cue [BL: $-3,500 \mathrm{~ms}-3,000 \mathrm{~ms}$ ] that was common to every TWOI anticipation phase (early, mid and late). This second analysis was conducted to enable comparison to previous studies (Brown, Seymour, Boyle, et al., 2008; Brown et al., 2014) that used the same baselining method, and to explore to what extent the results from the primary analysis were dependent on the baselining method used. All statistical analyses for the anticipation phase were adjusted for multiple comparisons.

All analyses for the post-stimulus phase TWOI [200 ms $600 \mathrm{~ms}$, centred on the LEP, were baseline corrected to $-500 \mathrm{~ms}$ prior to the laser stimulus [BL: $-500 \mathrm{~ms} 0 \mathrm{~ms}]$.

We also calculated the SD (standard deviation) of the EEG potential over trials for every time sample across the whole epoch [-3500 $1,500 \mathrm{~ms}$ ] and compared the results between the two groups to evaluate for possible differences in variability of the data over trials. This was to test whether any group differences found in ERP amplitudes and sources from the main analyses might have resulted from differences in data variability; such variability can arise from noise in the EEG signal (including motion and other artefact) rather than from neural signals. Such noise was expected to be greater in the PD group and therefore required assessing in order to interpret the results.

\section{Source localisation analysis parameters}

SPM12 EEG and MATLAB scripts were used to estimate the sources of the anticipation- and laser-evoked potentials using LORETA. The forward model was created using an 8196 vertex template cortical mesh coregistered to the electrode positions of the standard $10-20$ EEG system. A three-shell boundary element model (BEM) EEG head model available in SPM12 was used to compute the forward model. The images were smoothed with a $12 \mathrm{~mm}$ full width at half maximum (FWHM).

\subsubsection{EEG analysis statistical analysis}

For the analysis of the anticipatory TWOIs, a three-way repeated measures ANOVA was applied, with one between-subject factor of group (HC vs. PD) and two within-subject factors of certainty [known (low/high) v unknown] and expectation [low vs. high (known)]. For the analysis of the post-stimuli TWOI, a three-way repeated measures ANOVA was applied, with one between-subject factor of group (HC vs. PD) and two within-subject factors of certainty [known (low/high) vs. unknown] and intensity [low v high (known and unknown)].

Source localisation results were reported as follows. To control for multiple comparisons, a cluster-forming threshold of $p<.001$ was used and resulting clusters were considered significant at FWE $(p<.05)$. In addition, the clusters which did not reach significance after FWE, yet were significant at the uncorrected threshold, were also reported if the location of the clusters were within regions associated with anticipation. The inclusion of such source clusters is highlighted when reported. Significant clusters were also restricted to $>100$ voxels in size and regions labelled using the anatomical automatic labelling (AAL2) toolbox in SPM. We extracted the eigenvariate data from significant source estimates to investigate possible correlations with behavioural measures including MDS-UPDRS (PD group only), HADS, PCS, chronic pain maximum VAS score and laser voltage for high pain (V). The $p$ value was adjusted for multiple comparisons, $p<.01$. 


\section{RESULTS}

\subsection{Behavioural results}

\subsubsection{Questionnaires}

Group comparisons were carried out using a Mann-Whitney $U$ test of questionnaire scores. Our study did not show evidence of a significant difference between PD and HC groups reported in the PCS $(U=177.50, p=0.198)$ or $\operatorname{HADS}(U=212, p=0.660)$. The distributions of the questionnaire scores are shown in Figure 2. The OFF MDS-UPDRS-III motor score recorded in the PD group ranged from 15 to 78 , with a mean of $38.3 \pm 16.56$. For reference, a high MDS-UPDRS-III score indicates more severe movement impairments.

Fig. 2

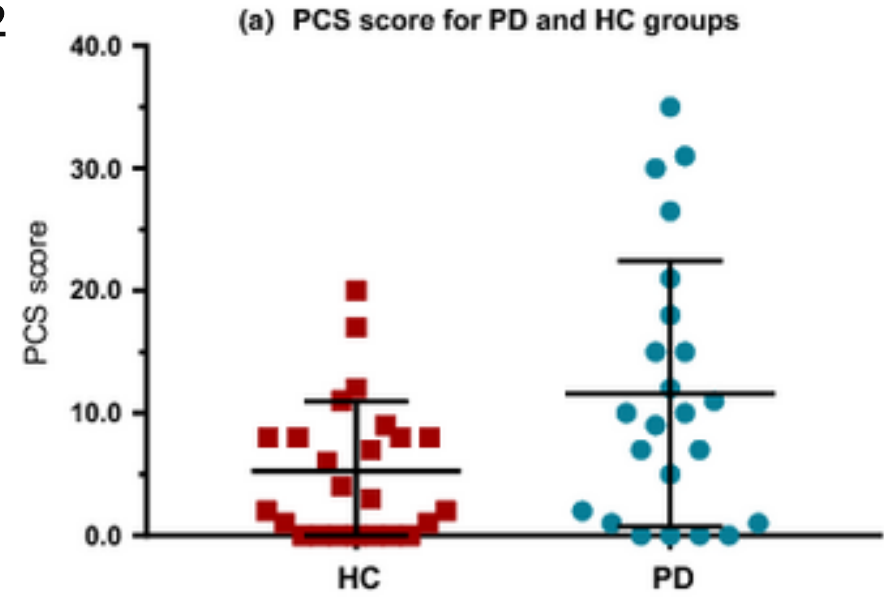

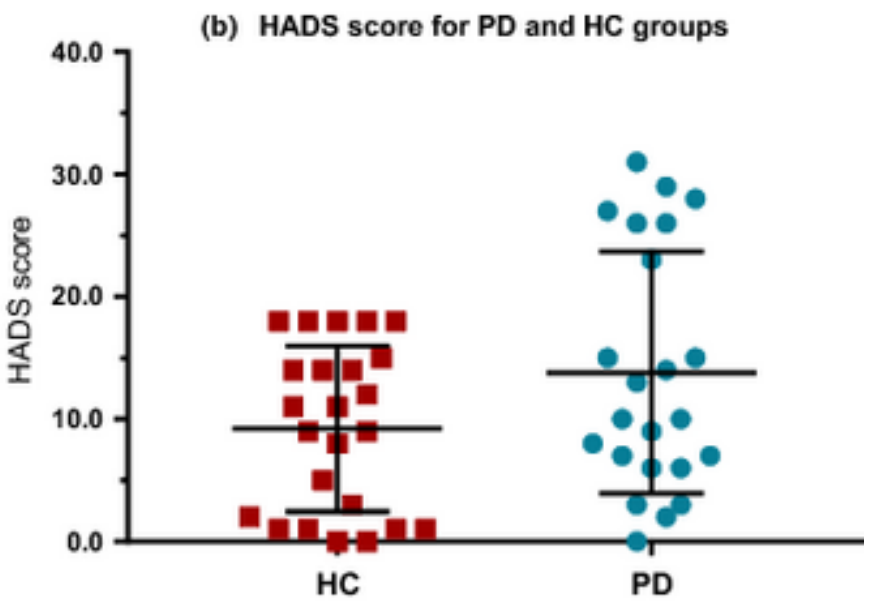

The individual scores for (a) Pain Catastrophizing Scale (PCS) and (b) Hospital Anxiety and Depression Scale (HADS). There was no evidence of a difference in either questionnaire between the two groups

\subsubsection{Laser behavioural results}

The psychophysics ramping procedure was used to calculate the participants' individual voltage level required to induce a high pain score. An independent sample $t$ test with a Welch's degrees of freedom correction was used to determine if there were differences between the HC and PD groups in the voltage required to induce a high pain score. There was a statistically significant difference in voltage required to induce a high pain between HC and PD (Figure 3), with PD requiring a lower voltage level ( $1.99 \pm 0.44 \mathrm{~V})$ compared to $\mathrm{HC}(2.20 \pm 0.25 \mathrm{~V}),(95 \% \mathrm{CI}, 0.21[0.003-0.432], t(34.75)=2.060, \mathrm{df}=34.75, p=0.047)$. To assess whether the severity of the PD participants' movement symptoms was correlated with individual pain tolerance, a correlation between the pain tolerance (measured as the voltage of the laser required to induce high pain) and the severity of movement impairment and muscle rigidity in the PD 
participants (MDS-UPDRS(iii)) was completed using Spearman's correlation and reported no significant correlation, $r_{\mathrm{s}}(43)=-0.177$, $p=0.257$ (see Figure 4).

Fig. 3

Laser voltage required to induce level 7 pain

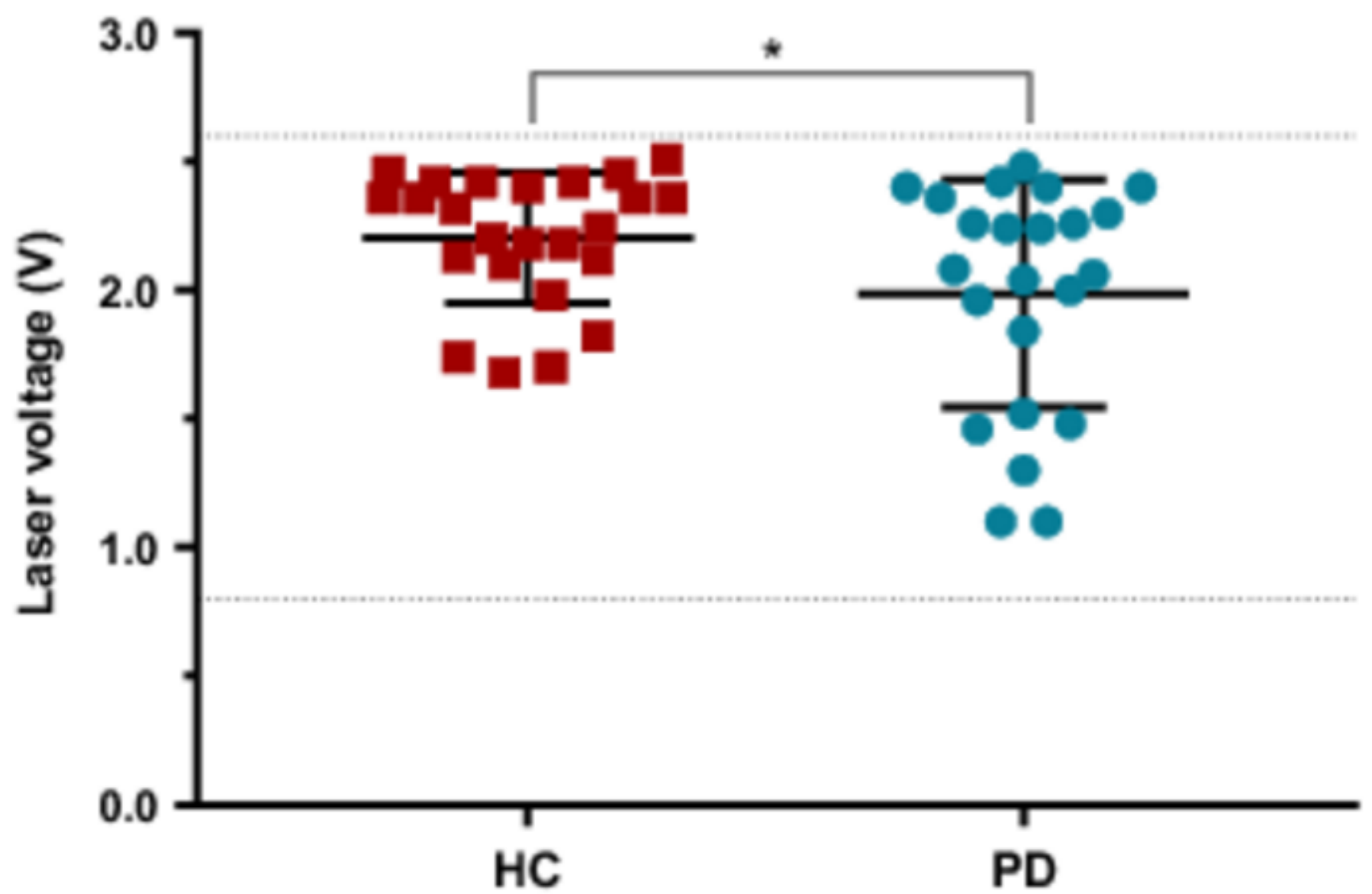

The voltage of the laser required to induce high (level 7) pain. The PD group required a lower voltage level compared to the $\mathrm{HC}$ group to induce the equivalent pain. Data are presented as a dot plot to display the individual results and show the spread of the data within each group. Laser voltage was delivered from $0.8 \mathrm{~V}$ to a maximum of $2.5 \mathrm{~V}$ in increments of $0.06 \mathrm{~V}$. ${ }^{*} p<.05$. HC, healthy control; PD, Parkinson's disease 
Fig. 4

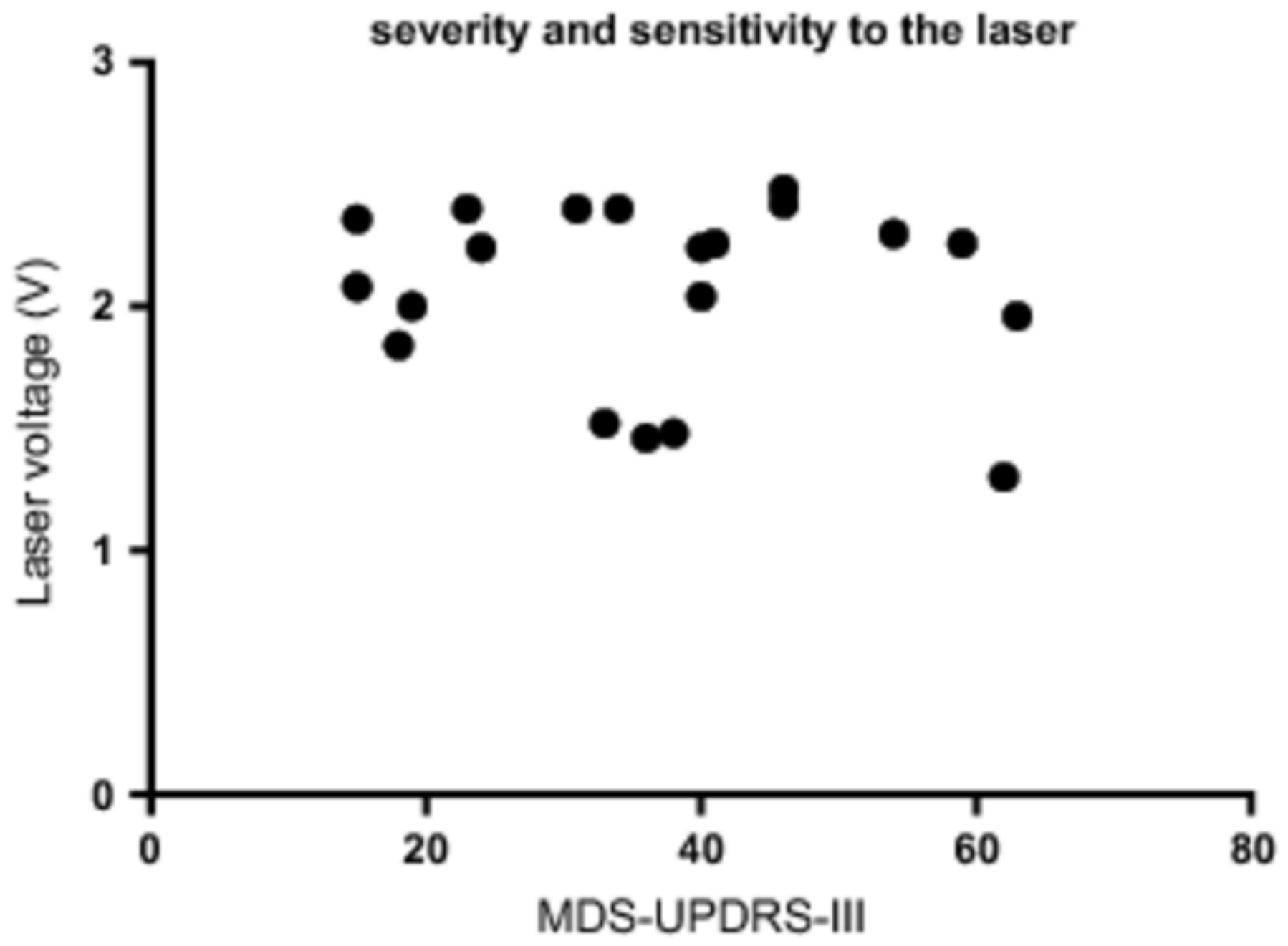

The PD group was assessed for a relationship between the severity of their movement impairment and their tolerance to the laser (measured as the voltage of the laser required to induce a level 7 pain). A Spearman's rank correlation did not show evidence of a relationship between the MDS-UPDRS(iii) and the laser voltage in the PD group 
The mean and standard error (SE) of the pain rating scores for all conditions were calculated: low (PD: $2.54 \pm 0.23, \mathrm{HC}: 2.0 \pm 0.18$ ), high (PD: $4.94 \pm 0.30$, HC: $4.7 \pm 0.26$ ), unknown low (PD: $3.06 \pm 0.25$, HC: $2.4 \pm 0.22$ ) and unknown high (PD $4.53 \pm 0.33$, HC: $4.1 \pm 0.26$ ). The pain rating scores for the laser stimuli during the main experiment were investigated using a three-way mixed ANOVA with a betweensubject factor of group and within-subject factors of certainty (known $v$ unknown) and intensity (low $v$ high). The data met homogeneity threshold $(p>0.05)$ calculated by the Levene's test of equality of variances. There was no effect of group $F(1,43)=2.347, p=.133$, $\eta^{2}=0.52$. There was an effect of intensity $F(1,43)=290.992, p=.000, \eta^{2}=0.871$, yet no effect of certainty $F(1,43)=0.006, p=.937$, $\eta^{2}=0.000$. However, there was a significant two-way interaction between certainty and intensity $F(1,43)=59.188, p=0.000, \eta^{2}=0.579$, such that the pain ratings of unknown low were higher $(+0.52 \mathrm{~V})$ compared to known low, whilst ratings of unknown high were lower $(-0.46 \mathrm{~V})$ compared to known high (see Figure 5). This result acts as a manipulation check that demonstrates that the experiment was successful in inducing expectations (via anticipation cues) that modulated pain in the expected direction as seen in previous research (Brown, Seymour, El-Deredy, et al., 2008). The study did not find evidence of a significant difference in the effect of intensity or certainty on pain ratings between the two groups (HC vs. PD), nor was there any interaction between intensity, certainty and group. 
Fig. 5

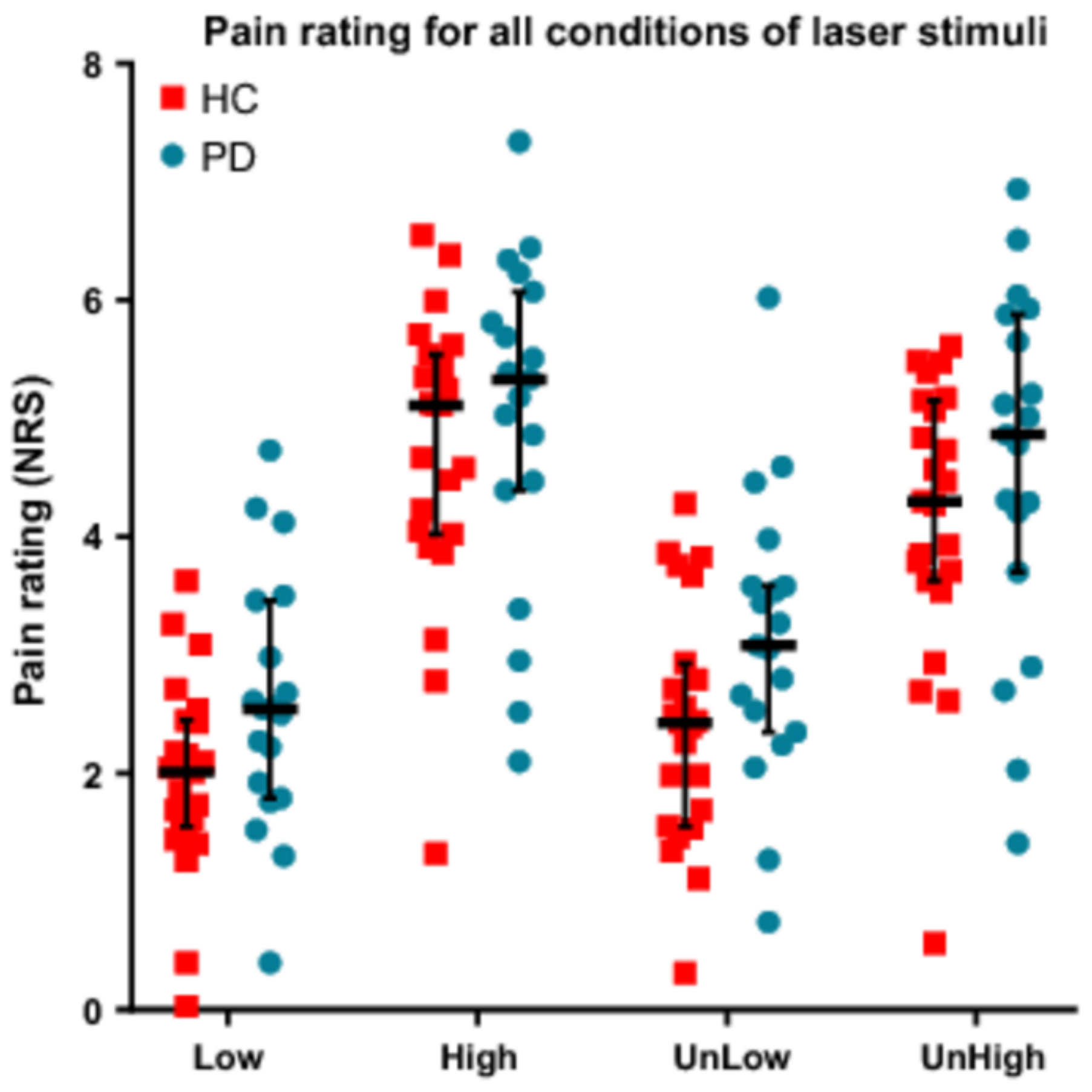


The pain rating for each laser condition is displayed for the HC and PD groups. There was a significant interaction between certainty and intensity such that unknown low was rated higher than known low, whilst unknown high was rated lower than known high. This result was shown in both the HC and PD groups. UnLow, 'Unknown' Low; UnHigh, 'Unknown' High; HC, healthy controls; PD, Parkinson's disease; NRS, Number Rating Scale

\subsection{EEG results}

\subsubsection{Scalp-level Whole-head analysis}

SPM whole-head statistical analysis of the early $[-2,500-2,000 \mathrm{~ms}]$, mid [-1,500 $-1,000 \mathrm{~ms}]$-and late [-500 0 ms] anticipatory phases reported no evidence of a significant difference of group, certainty or expectation. This was true for both baseline methods (see Methods section). Similarly, the post-stimulus TWOIs [200 $600 \mathrm{~ms}$ ] reported no significant group or certainty differences. The intensity factor in the post-stimulus TWOI reported increased activity in high pain condition compared to low pain (SPM results reported in Table S1).

The anticipatory response over time was calculated for each participant and displayed as topography plots in Figure 6 and highlights the variability in sensor-level response within each group. The average response for mid- and late anticipation for the HC and PD groups is shown in Figure 7. In addition, the difference between HC and PD group was also determined by subtracting the HC amplitudes from the PD data and shown in Figure 7. Despite the SPM scalp-level analysis not reporting a significant difference, an anticipation response is seen in the central scalp region which is more prominent in PD than HC. Because scalp responses are a summation of deeper brain events from different sources, the scalp-level analysis was hence followed up by source localisation analysis. 
Fig. 6

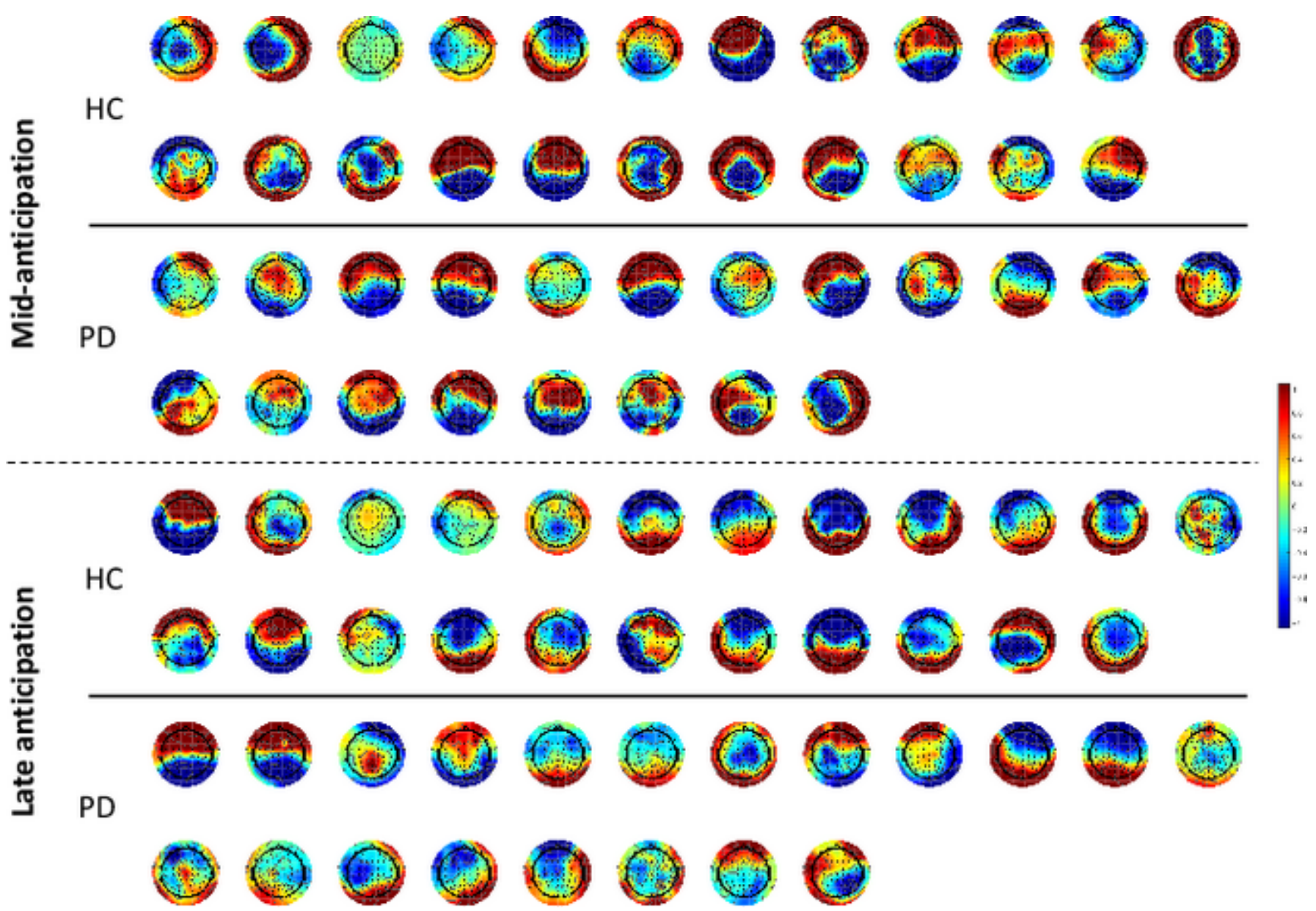

The sensor-level neural response was averaged across time for each channel and plotted as topoplots. An individual topography is shown for the average response for mid- $[-1500-1000 \mathrm{~ms}]$ and late $[-5000 \mathrm{~ms}]$ anticipation time windows for HC and PD. Mid-anticipation is baseline corrected to $[-2,500-2,000 \mathrm{~ms}]$ and late anticipation to $[-1,500-1,000 \mathrm{~ms}]$. The scale is set at -1 to $1 \mu \mathrm{V}$ for all plots

Fig. 7

\section{Anticipation}

\section{naid \\ 1 ato}




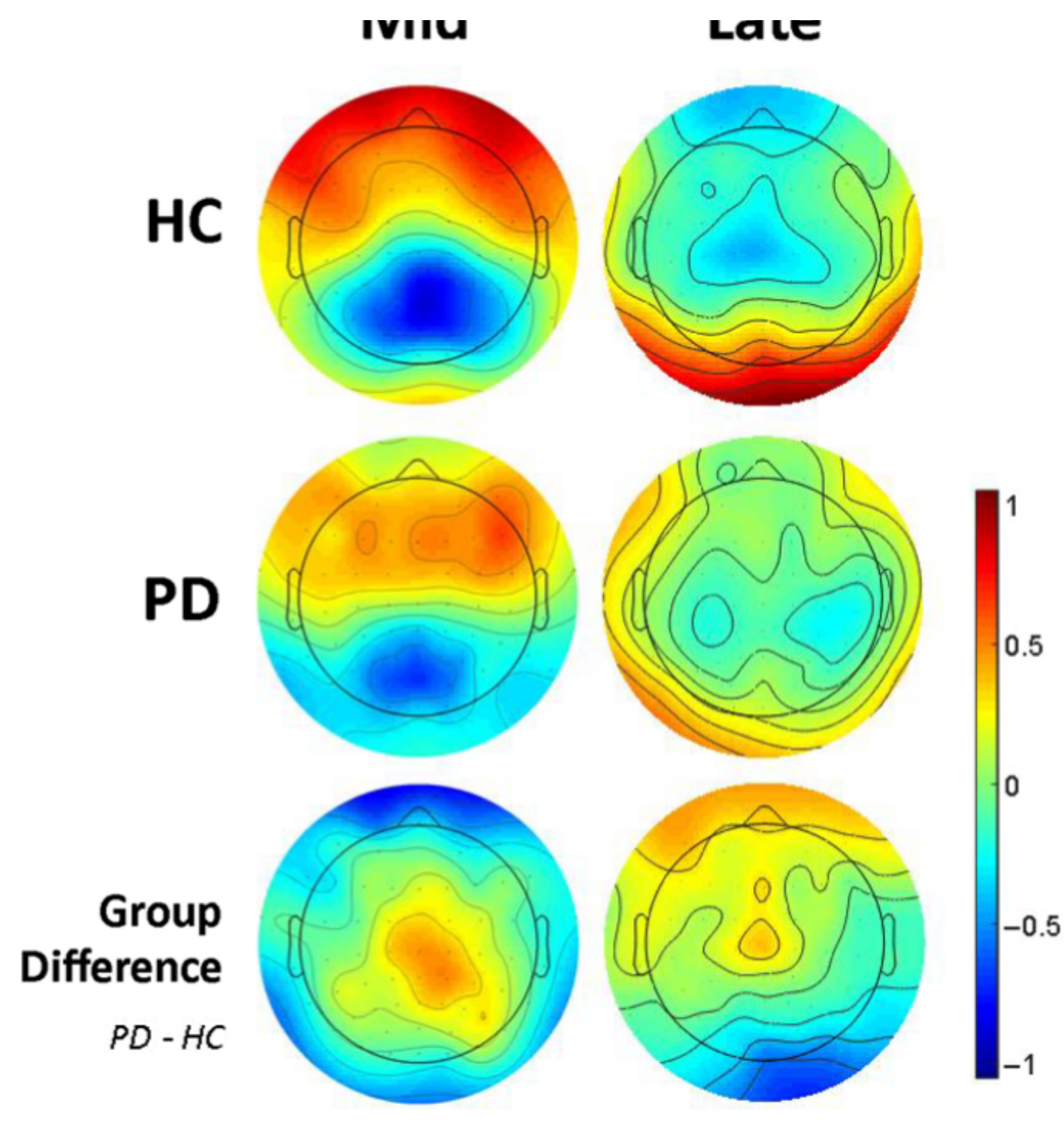


Topography plots showing the average response for the mid- and late anticipation time windows for HC and PD. Midanticipation is baseline corrected to $[-2,500-2,000 \mathrm{~ms}]$ and late anticipation to $[-1,500-1,000 \mathrm{~ms}]$. The difference between the PD and the HC group was calculated via subtracting the HC data from the PD data. The same scale has been used for all topography plots. In the group difference plot, the red (1) highlights a more positive response in the PD participants, whilst blue (-1) indicates a more negative amplitude in PD

AQ6

AQ7

\subsubsection{Source EEG analysis}

\section{Group differences}

Source localisation analysis using the distinct baselines prior to the auditory cues revealed that the PD group showed a higher degree of activity compared to the $\mathrm{HC}$ group during mid- and late anticipation phases (see Figure 8). The F- and T-contrasts for group difference in each anticipation time window are outlined in Table 1. The clusters which are denoted with ' $\diamond$ ' in Table 1 were used for correlation analysis with subsequent behavioural parameters. Source clusters were also reported at the uncorrected level if they were within regions previously associated with anticipation including the hippocampus (Reicherts et al., 2017), the postcentral gyrus (Yang, Jackson, \& Huang, 2016) and the cingulate cortex (Shackman et al., 2011). Hence, there is evidence for a potential difference within the early anticipation between the two groups. The difference in neural activity between the two groups is shown in Figure 9 which displays the eigenvariate values for all clusters outlined in Table 1 for HC and PD groups.

Fig. 8

\section{(a) Mid-anticipation [-1,500-1,000 ms]}

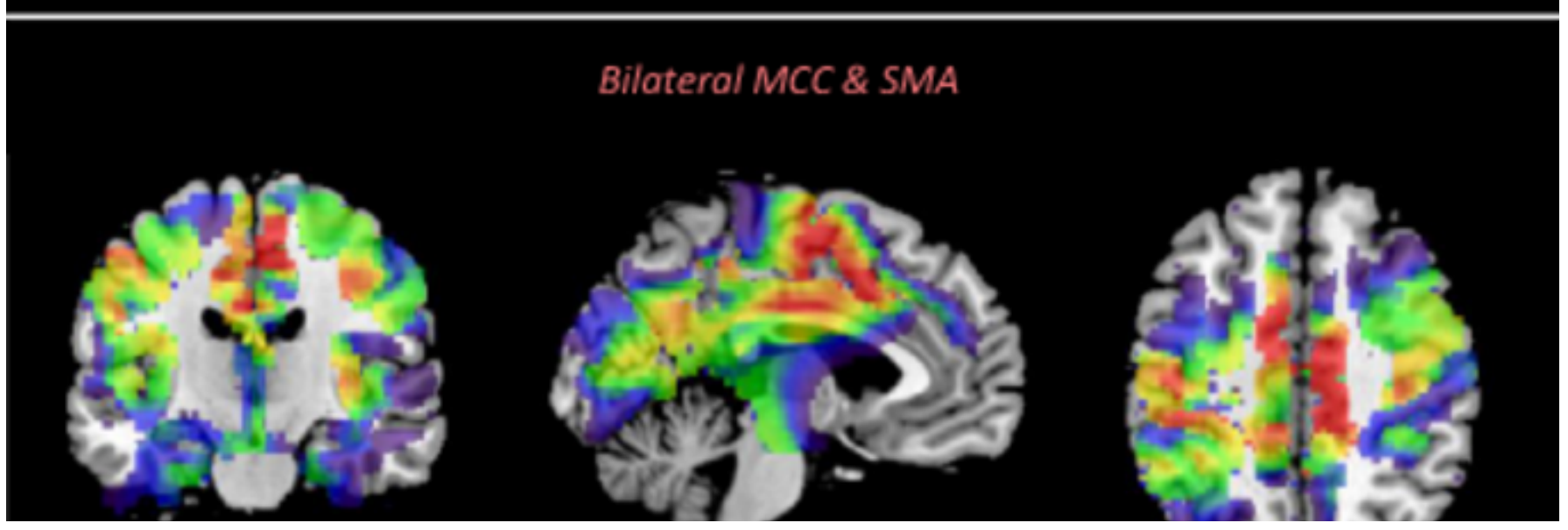




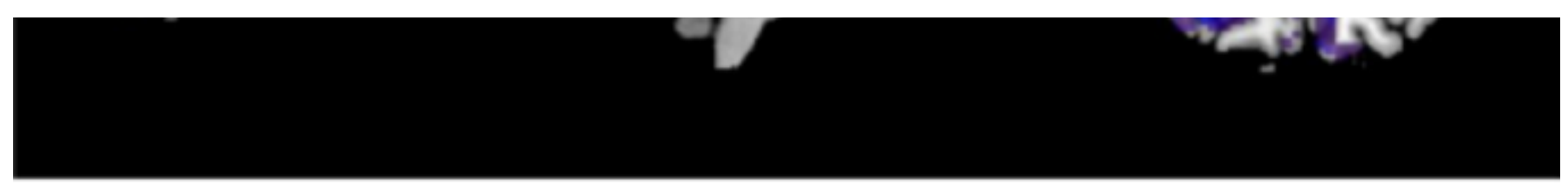

\section{(b) Late anticipation [-500 $0 \mathrm{~ms}]$}
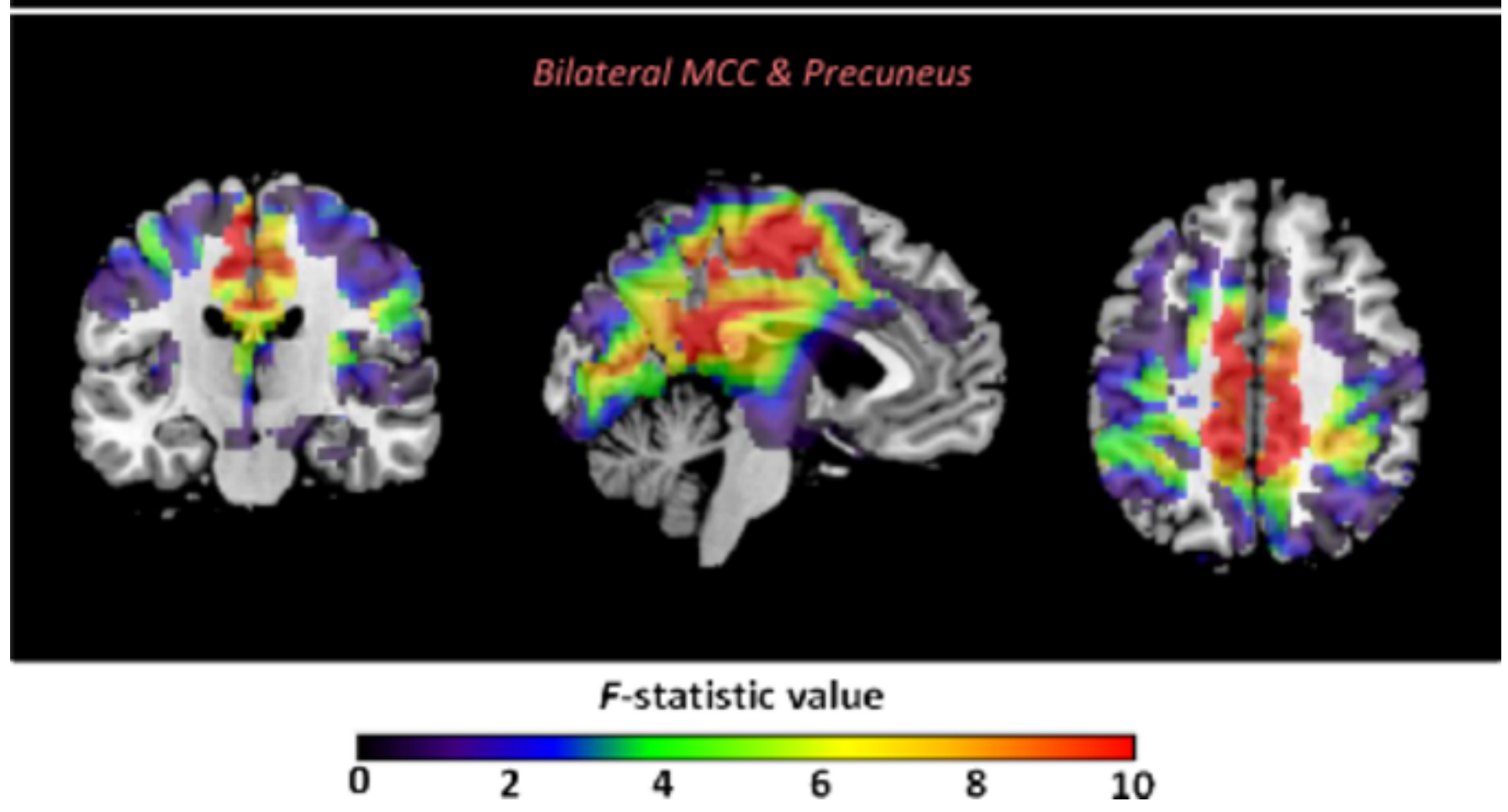

Source estimates for significant F-contrasts using MRIcroN software. Data are presented non-thresholded and presented as F-statistic, from low to high. The red areas highlight the regions where the PD group showed evidence of a statistically significant increased activation compared to the HC group during anticipation to the noxious stimuli. Panel (a) shows the increased activation in the PD group during the mid-anticipation phase $[-1,500-1,000 \mathrm{~ms}]$ and is baselined to $[-2,500$ $-2,000 \mathrm{~ms}$. The PD group showed increased activation in the bilateral midcingulate cortex (MCC) and supplementary motor area (SMA). Panel (b) shows the increased activity within the PD group during the late anticipation phase [-500 $0 \mathrm{~ms}]$ and is baselined to $[-1,500-1,000 \mathrm{~ms}]$. The regions of increased activity included the bilateral MCC and precuneus 
Table 1 Group effect on sources of early, mid- and late anticipation-evoked responses

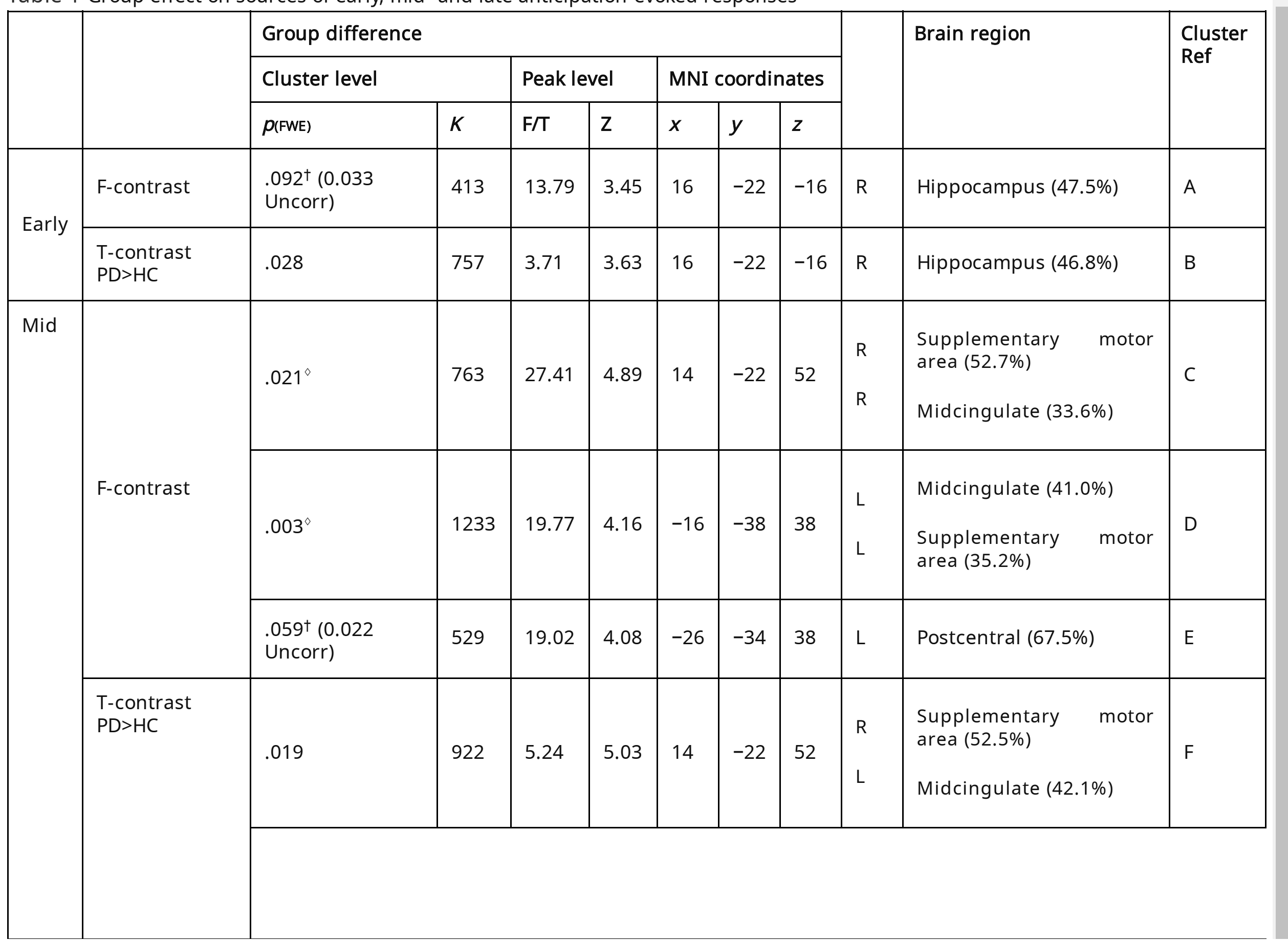




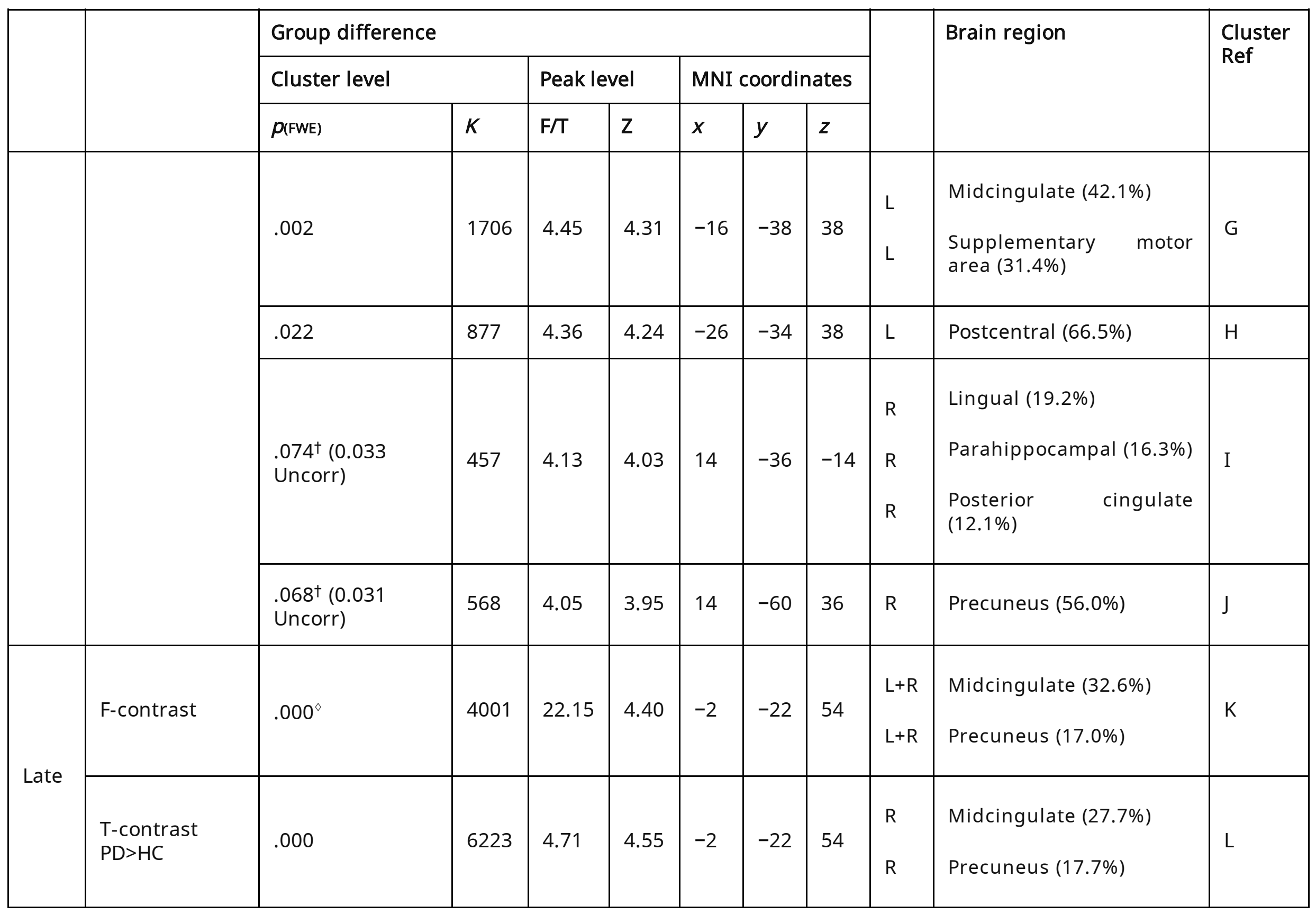


A threshold of clusters $>100$ voxels was set and results were restricted to FWE correction and the $p$ value adjusted for multiple comparisons $(p<.025)$. Results labelled with ' $\diamond$ ' met these criteria and are used for Figure 8 and correlation analysis (Figures 11 and 12). In addition, clusters are also reported for results which did not show significance after FWE, yet showed significance at the uncorrected (Uncorr) threshold and were within regions which have previously been associated with anticipation. These clusters are signified with $\dagger$, and both FWE and uncorrected $p$ values are shown. Each cluster has been assigned a letter for reference in other figures. Figure 9 displays the eigenvariate values extracted for each cluster (A-L) at the F- and T-contrasts to show the distribution within the HC and PD groups. The anatomical automatic labelling (AAL2) atlas was used to report the regions within the significant cluster for the group effect. The region with the highest percentage overlap is shown, unless an equivalent share of percentage overlap was seen. A label of 'Unknown' was not reported. The full report of all of the percentage overlaps can be seen in the Table S3.

Fig. 9

F-Contrast

T-Contrast

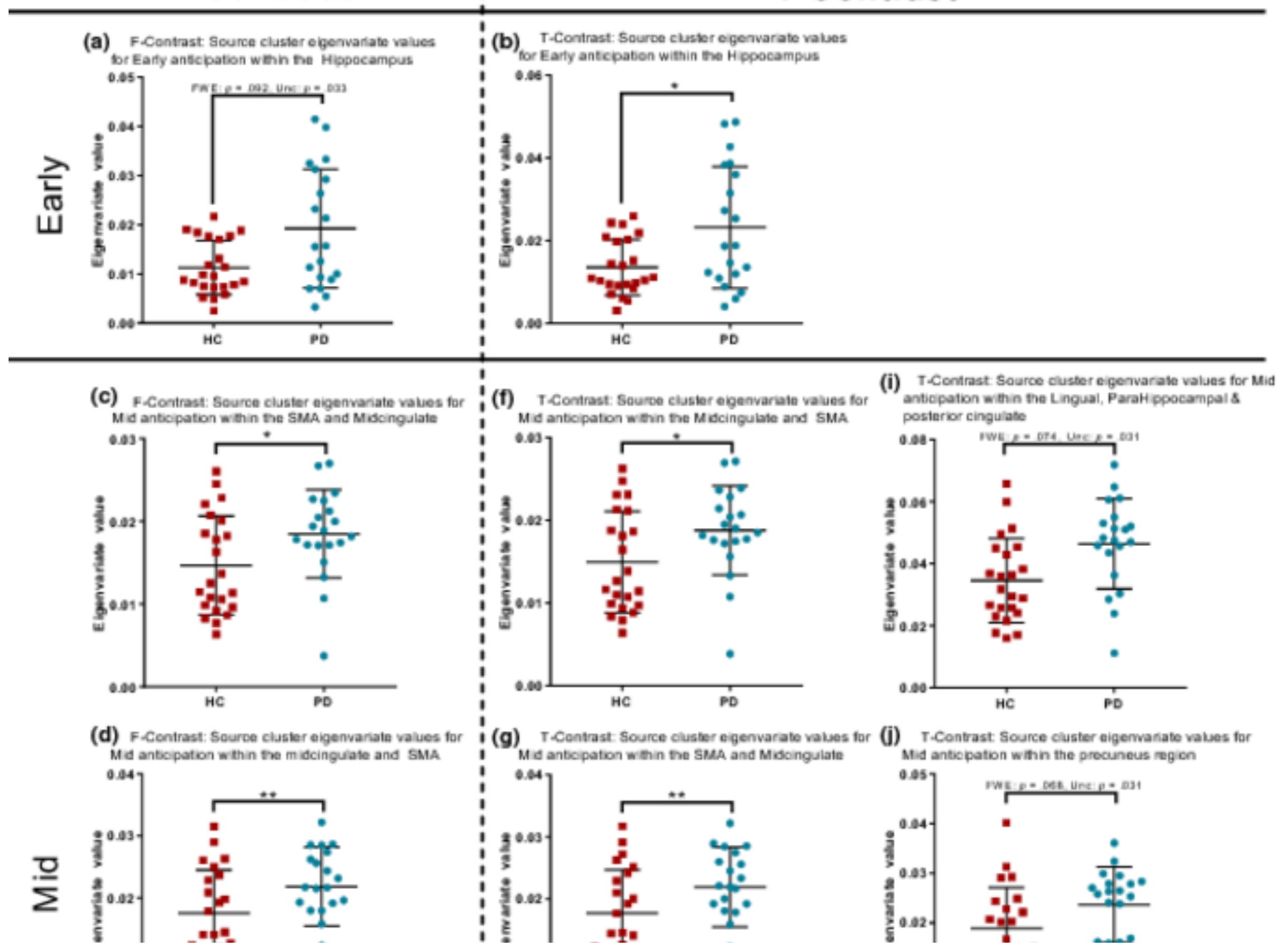



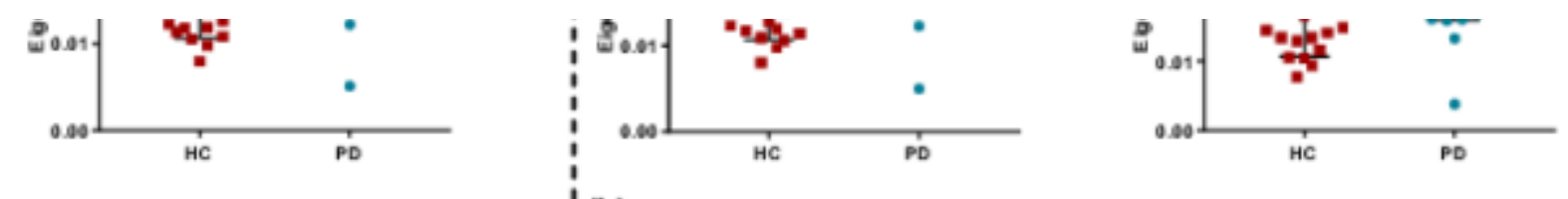

(e) F.Contrast: Source ciuster eigenvariate values

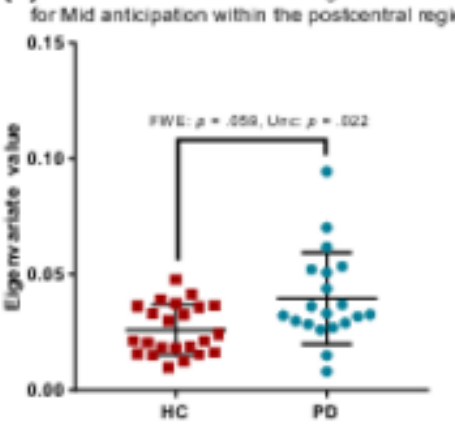

(h) T-Contrast: Souroe cluster eigenvariale values for

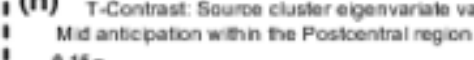

${ }^{0.15}$ ]

$\frac{3}{3} 0.10-\sqrt{\square}$

产

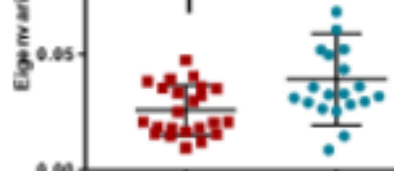

PD

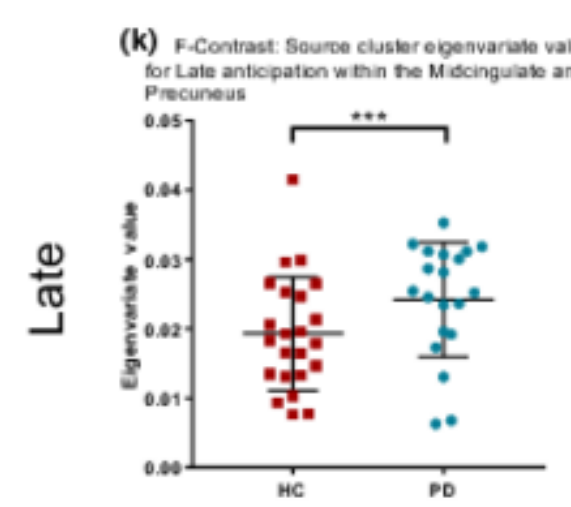

\section{(I) T-Contrast: Souroe elustor egenvarato values \\ for Late anticipaton within the Midcingulate and}

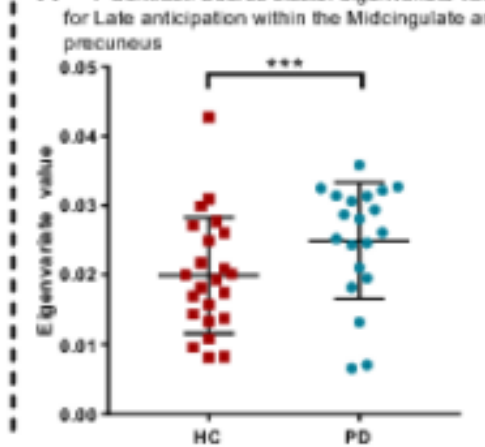

Following source localisation, the eigenvariate values were extracted from all clusters which showed group differences between HC and PD outlined in Table 1. The eigenvariate values are plotted for the HC and PD groups and reported with group mean and standard deviation error bars. Significance is highlighted for each comparison such that $* p<0.05$, ${ }^{*} p<0.01,{ }^{* *} p<0.001$. The significant uncorrected $p$ value is reported for results which did not reach significance at FWE

During early anticipation, there was possible evidence that the presentation of the 'Unknown' visual cue induced a higher degree of activity in comparison with the known conditions of 'Low' and 'High'. The F-contrast cluster did not reach FWE significance ( $p=.053$, $F=19.87, k E=520)$; however, the uncorrected $p$ value of 0.019 and visual inspection of the eigenvariate value distribution within each group (Figure 10) warranted follow-up t tests. The T-contrast (unknown>known: $p=0.013, T=4.32, k E=967$ ), with peak voxel at $x .48 y .16$ $z:-16(\mathrm{~mm})$ indicated a higher activity during the unknown condition in contrast to the known condition. The cluster is within the right 
superior temporal pole (37.5\%), right mid-temporal pole (18.3\%), right superior temporal (14.6\%) and the right insula (3.7\%). Contrasts for expectation (low $v$ high) during anticipation, and certainty (known $v$ unknown) within the post-stimulus TWOI, did not show evidence of significant differences in source estimates. During the post-stimuli TWOI, there was a significant difference in the intensity, such that a greater response was seen in the high intensity condition compared to low (see Table S2).

Fig. 10

\section{F-Contrast: Source cluster eigenvariate values for Early}

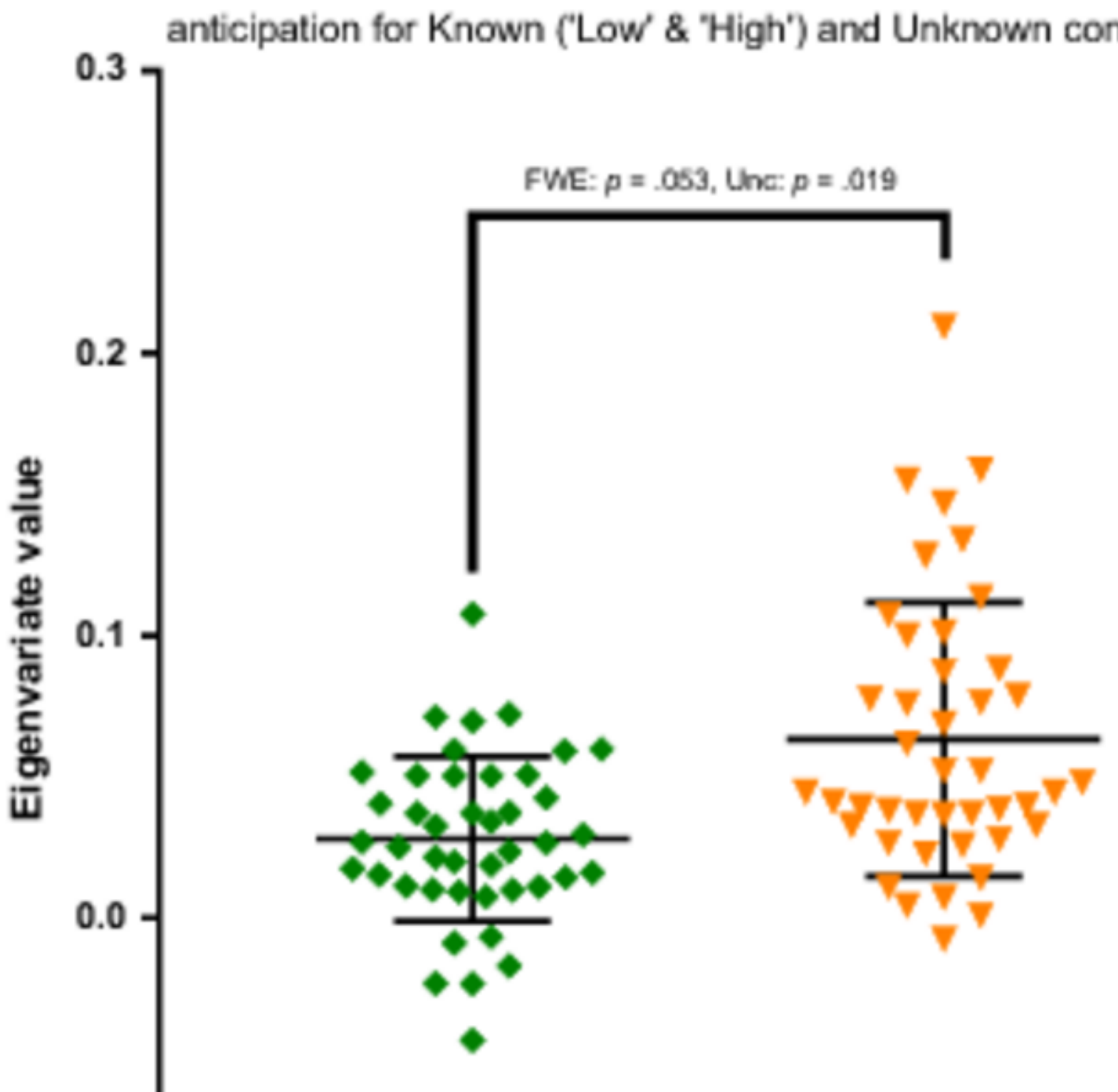




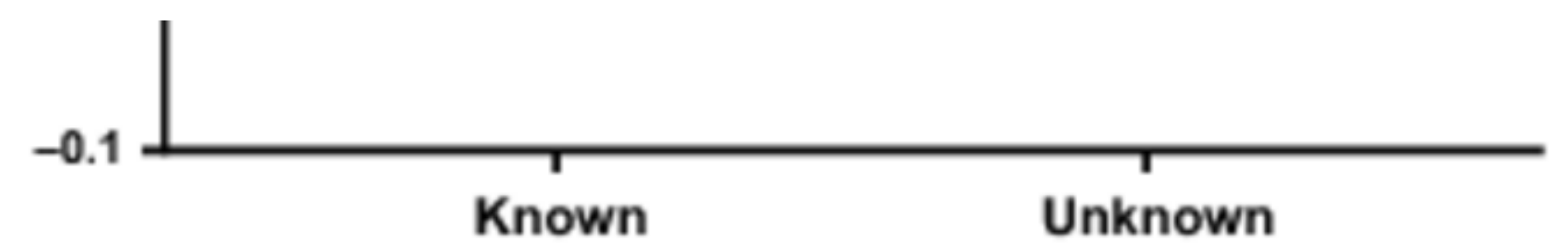

Source localisation analysis indicated that the presentation of an unknown cue induced a higher level of neural activity during early anticipation in comparison with the known cues of 'Low' and 'High'. The cluster was located within the right superior temporal pole, mid-temporal pole, superior temporal region and insula. The effect of certainty on neural activity did not reach significance at FWE $(p=0.053)$. Nevertheless, the uncorrected $p$ value $(0.019)$ in combination with the higher eigenvariate values for the unknown condition shown in the dot plot indicates that a difference is likely

\section{Source correlations with behavioural measures}

The eigenvariate data from the source estimates which reached significance after FWE correction (denoted with ' $\diamond$ in Table 1) for the mid- and late anticipation group effects were extracted for correlation analysis. Spearman rank correlation of mid-anticipation clusters (Table 1: clusters C and D) and the late anticipation cluster (Table 1: cluster K) were investigated for correlation with MDS-UPDRS motor score (PD only), HADS, PCS, maximum chronic VAS score (PD only), the voltage of the laser and pain rating. The distributions of the data are shown in Figures 11 and 12. The $p$ value was adjusted to account for multiple comparison, and no significant correlations were highlighted. 
Fig. 11 Scatterplots for Behavioural results versus Mid Anticipation neural activity extracted from source clusters

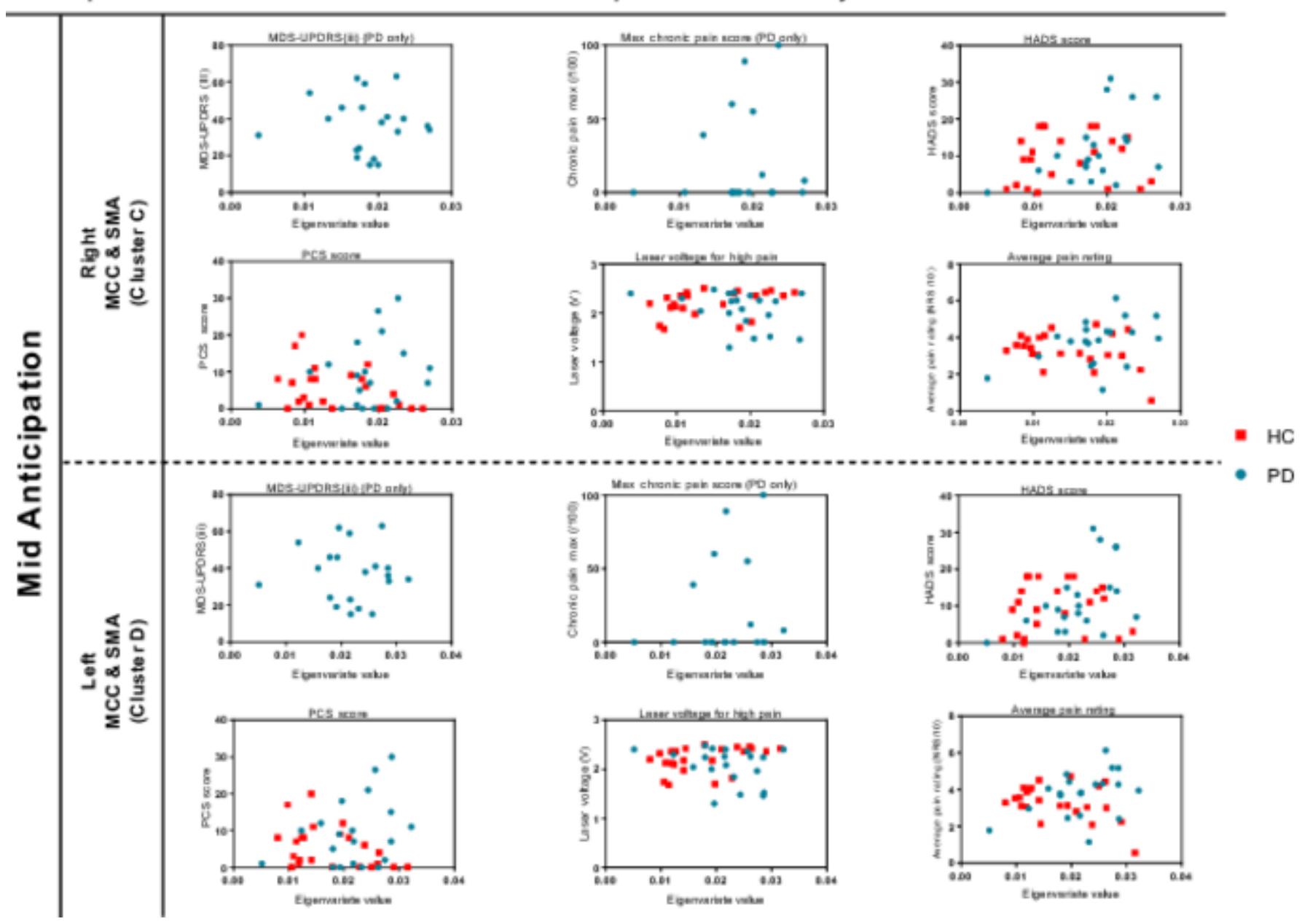

Scatterplots of the relationships between the anticipatory neural activity and the behavioural results. Following source localisation, the eigenvariate values were extracted from clusters which showed a significant group difference at FWE correction (see Table 1). Within mid-anticipation, two clusters located within the MCC and SMA (C and D) were investigated for correlations between the behavioural factors; MDS-UPDRS(iii) (PD only), max chronic pain (PD only), HADS, PCS, laser voltage for high pain and average pain rating. Spearman rank correlation analysis did not report any significant correlations 
Fig. 12 scatterplots for Behavioural results versus Late Anticipation neural activity extracted from source clusters

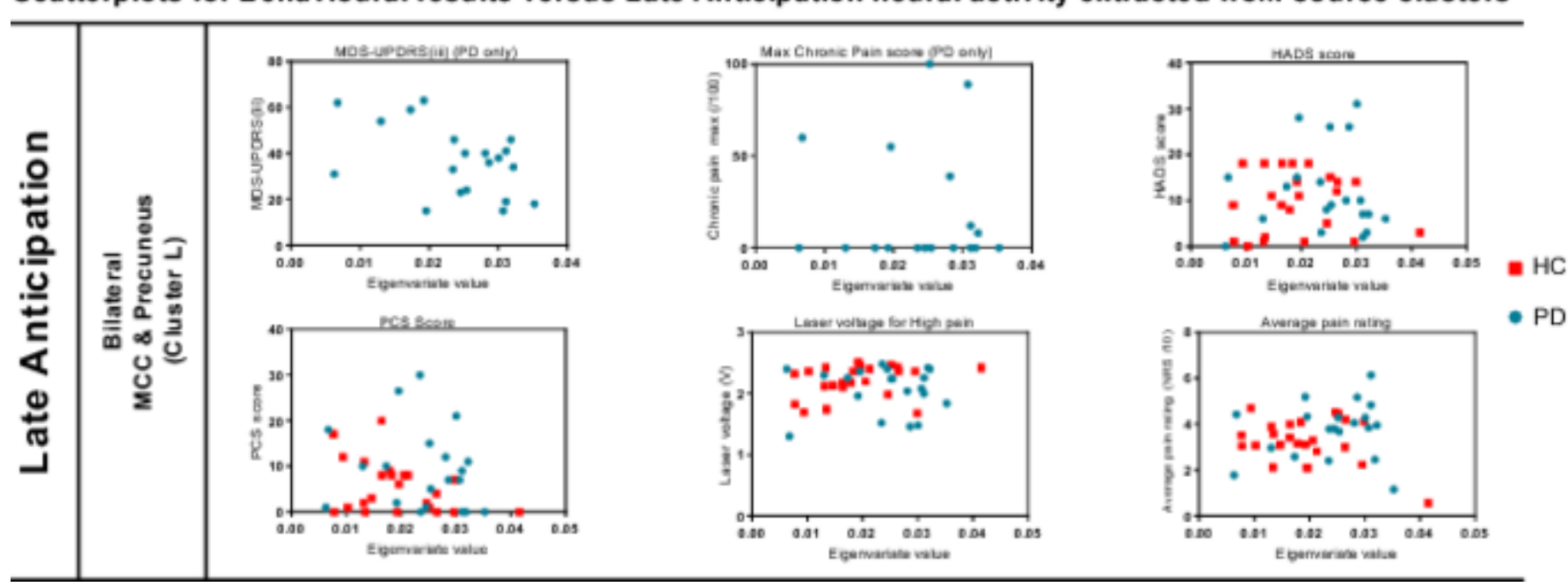

Scatterplots of the relationships between the anticipatory neural activity and the behavioural results. Following source localisation, the eigenvariate values were extracted from clusters which showed a significant group difference at FWE correction (see Table 1). Within late anticipation, one cluster within the MCC and precuneus (cluster K), was investigated for correlations between the behavioural factors; MDS-UPDRS(iii) (PD only), max chronic pain (PD only), HADS, PCS, laser voltage for high pain and average pain rating. Spearman rank correlation analysis did not report any significant correlations

\subsubsection{Standard deviation analysis}

The analysis of the SD-over-trials across the whole epoch at sensor-level EEG revealed no significant differences between the HC and PD groups, nor within-subject contrasts, certainty or expectation.

\section{DISCUSSION}

In the present study, we used EEG source localisation techniques to investigate the pain processing in PwPD in the practically defined OFF state (after $12 \mathrm{hr}$ of withdrawal of anti-Parkinsonian medication). There were three main findings. Firstly, the PD group were more sensitive to the laser and required a lower voltage level to evoke painful stimuli. Secondly, EEG source localisation showed that the PD group had a heightened anticipatory activity during the anticipation phase prior to acute heat pain. And finally, the heightened anticipatory response was independent of PD motor severity (MDS-UPDRS), mood (HADS), pain coping mechanisms (PCS) and severity of chronic pain (max chronic VAS score). This present study provides evidence to support the hypothesis that there is an augmented topdown processing during the anticipation of pain in PwPD which may help us to understand the high prevalence of pain in PwPD. 


\subsection{Pain tolerance}

The pain tolerance of the participants was assessed via the voltage required of the laser required to induce a level 7 pain. We observed that overall the PD group required a lower voltage and were hence more sensitive to the laser than the HC group. This difference was potentially driven by a subgroup of six PD individuals whom required a voltage that was lower than the lowest HC value. To establish whether their sensitivity to the laser corresponded with similarities in neural responses, we looked at their scalp-level topography and extracted eigenvariate values from source estimates for the anticipatory time windows. We did not identify any connecting characteristics within the PD subgroup that would define them as a distinct subgroup of PD.

\subsection{Altered top-down control}

The amplified anticipatory response seen in the PD group is evidence of altered top-down modulation prior to the nociceptive information reaching the brain. This opens the possibility that the heightened pain sensitivity in PD is not solely due to impaired peripheral, bottom-up, factors, but may also be due to the modulation within the brain.

The significance of altered top-down processing is well established in the pain field: different attentional states can directly and substantially alter pain intensity and unpleasantness (Miron, Duncan, \& Bushnell, 1989); anxious anticipation of aversive stimuli activates regions associated with pain perception, resulting in altered perception of the stimuli (Reicherts et al., 2017). Furthermore, the degree of anticipation of a noxious stimulus has been shown to directly correlate with the perceived stimuli intensity (Fairhurst, Wiech, Dunckley, \& Tracey, 2007; Pfingsten et al., 2001), and abnormal anticipation of a painful stimulus has been shown in a number of chronic pain conditions such as fibromyalgia (Brown et al., 2014; Burgmer et al., 2011; González-Roldán et al., 2016).

Here, we have shown increased cortical activation in the PD group during the anticipation of painful stimuli, including the midcingulate cortex (MCC), supplementary motor area (SMA) and precuneus. Importantly, the group effects seen during anticipation were independent of the MDS-UPDRS, PCS and HADS emphasising that it is likely that the underlying PD pathological process is causing the greater anticipatory activity rather than the consequence of motor impairment, coping mechanisms and mood state. The fact that the abnormal processing is independent of the MDS-UPDRS supports the hypothesis reported in Silverdale et al., 2018 that the high prevalence of chronic pain in PD is independent of the severity of the motor impairments and could be due to top-down modulation of the nociceptive information.

Our results are consistent with a single previous fMRI study, using a very different protocol which demonstrated that PwPD showed a significantly reduced activation within the inhibitory descending pain pathway during the anticipation of pain and an increased activation within the MCC during pain perception (Forkmann et al., 2017). In combination with the results presented in this current study, and evidence which has shown similar altered anticipatory processing in chronic pain conditions (e.g. FM), we reason that PwPD display maladaptive top-down modulation during pain processing which may help to explain the high prevalence of pain within the PD community. However, a definite conclusion of a causal link between altered anticipatory processing in PD and chronic pain prevalence cannot be confirmed without further investigations. 


\subsection{Regions activated during the anticipatory response}

The location of the amplified anticipatory signal within the PD group during mid- and late anticipation was mainly, but not limited to, the SMA and the MCC. The SMA and MCC have a role in the selection and planning of movements (Morecraft \& Van Hoesen, 1998; Rushworth, Walton, Kennerley, \& Bannerman, 2004; Russo, Backus, Ye, \& Crutcher, 2002), and are indicated to be involved in the prediction of aversive events and the movement response required (Morrison, Peelen, \& Downing, 2007). The SMA and the MCC have shown overlapping functional connectivity during pain processing and motor control (Misra \& Coombes, 2015), and enhanced activation within the SMA has been associated with patients with phantom limb pain (Dettmers et al., 2001).

The MCC is a region involved in cognitive processing and is associated with the fear response, pain processing and specific motor outcomes to painful stimuli (Shackman et al., 2011; Vogt, Berger, \& Derbyshire, 2003). Abnormal connectivity within the MCC is associated with patients with migraines (Hubbard et al., 2014), and a heightened response within the MCC has been reported in PwPD during pain perception (Forkmann et al., 2017). In addition, in an extensive rodent study, the MCC showed to be central to the development of pain hypersensitivity in the absence of peripheral noxious stimuli (Tan et al., 2017). The study also demonstrated that silencing the MCC via optogenetic techniques partially reversed inflammatory hypersensitivity, thus highlighting the role of MCC in the development of chronic pain conditions (Tan et al., 2017).

Additionally, the MCC is also associated with reward processing (Hayden, Nair, McCoy, \& Platt, 2008; Pearson, Hayden, Raghavachari, \& Platt, 2009; Shackman et al., 2011), a process highly dependent on dopaminergic signalling and hence may be important due to the depletion of dopamine seen in PD. Abnormal reward processing is a possible predictor of the development of increased pain sensitivity (Nees \& Becker, 2017; Nees et al., 2017) and provides a potential explanation of why abnormalities in the MCC during pain perception are present in PwPD.

Thus, the MCC and SMA are important for the processing of nociceptive information and provide a putative anatomical substrate for enhanced top-down modulation of incoming nociceptive information.

\subsection{Laser-evoked potential}

The LEP was not significantly different between the two groups, yet did show a significant difference between low and high laser stimuli. The subjective experience of pain was standardised in both groups, such that participants experienced what they subjectively considered to be a low and high pain. We therefore did not expect a group difference in the LEP. The relationship between LEP and behavioural response has been reported to be highly variable and thus is not an accurate characteristic of pain processing to draw conclusions from (Iannetti, Hughes, Lee, \& Mouraux, 2008; Mouraux \& Iannetti, 2009).

Although research has previously shown that an altered degree of anticipation can modulate the LEP amplitude and pain unpleasantness, there is research by Clark et al., 2008 which reported that the characteristics of the LEP, specifically the P2 peak, were not affected by the duration of the anticipation period, nor correlated to the behavioural pain ratings. They concluded that the 
anticipatory response is more closely aligned with attentional processing rather than intensity coding of the stimulus. These findings are consistent with the results from this study and hence help to explain of how a difference during the anticipatory period did not affect the LEP response.

\subsection{Considerations of interpretation}

The MCC and SMA are regions associated with nociceptive processing; however, their activation is not unique to nociceptive processing. The regions are also associated with the processing of non-nociceptive salient stimuli (Mouraux, Diukova, Lee, Wise, \& Iannetti, 2011) and highlight that the cortical activity may not be unique to nociception. Therefore, a limitation in our interpretation is that the study did not include non-painful control stimuli. This would have allowed us to establish whether the abnormal anticipation response in PD patients is unique to pain anticipation. We would therefore recommend further investigation using non-painful and non-salient stimuli to further understand the abnormality in the PD cortical pain processing.

The present study has used source localisation to successfully quantify the anatomical origin of the activity recorded at scalp electrodes. These differences were not directly correlated to significant changes at scalp-level EEG and thus need to be considered when interpreting these results. It is known that there is no simple relationship between scalp and source activity, as a single electrode summates the potentials generated within neural sources and is dependent on the combination of the orientation, strengths and location of these potentials. Hence, as scalp topographies are the product of the addition of multiple brain sources, it is difficult to interpret them directly in relation to source localisation estimates. The comparison of SD-over-trials between the groups did not report any differences and indicates that group differences in data variability (e.g. due to noise) are unlikely to be a factor driving group effects at the source level. The source localisation results in this study are supported by conclusions drawn in Forkmann et al., 2017, which also showed increased activity within the cingulate cortex during pain perception in PwPD. Hence, we are confident that despite not seeing scalp-level group differences, the significant differences reported via source localisation are valid.

\subsection{Clinical impact}

The current perception of pain in PWPD is often considered to be a consequence of peripheral symptoms such as rigidity and stooped posture. However, our findings suggest the possibility that the treatment for chronic pain in PD could beneficially incorporate alternative treatments such as mindfulness and cognitive behavioural therapy (CBT). These treatments have been shown to reduce anticipation to noxious stimuli (Brown \& Jones, 2010), improve cognitive control of pain over time and reduce the severity of chronic pain (Kabat-Zinn, 1982; Kabat-Zinn, Lipworth, \& Burney, 1985). Nevertheless, further investigations are essential to establish whether cognitive therapies are a suitable treatment for pain in Parkinson's disease.

\section{CONCLUSION}

In conclusion, Parkinson's patients demonstrated enhanced anticipatory activity within the brain before an acute pain stimulus. Although we cannot conclude a causal link between altered anticipation in Parkinson's and the prevalence of chronic pain in PD, we have provided evidence for altered top-down processing of pain in Parkinson's disease, increasing the evidence for abnormal central processing in Parkinson's and other chronic pain conditions. Our results contribute to the building knowledge of the relationship 
between chronic pain and Parkinson's disease, and inform a possible scientific rational for novel treatment strategies in Parkinson's pain, including mindfulness, cognitive therapies and other treatments targeted at reducing top-down processing of pain.

\section{ACKNOWLEDGEMENTS}

The authors would like to thank Timothy Rainey who helped with data collection, and Stuart Watson, Donald Allan and Prawin Samraj from Medical Physics at Salford Royal NHS Foundation Trust for their technical support during the study.

\section{CONFLICTS OF INTEREST}

There are no conflicts of interest.

\section{AUTHOR CONTRIBUTIONS}

The study was designed by M.A.S., A.KP.J, C.A.B and S.L.M. The data were collected and analysed by S.L.M, with EEG analysis support from C.A.B. Patient recruitment was carried out by M.A.S, C.K and S.L.M. Statistical analysis and interpretation of results were carried out by all authors. S.L.M wrote the manuscript; all co-authors contributed to editing the manuscript.

\section{DATA ACCESSIBILITY}

Raw data, statistical output and analysis scripts will be accessible via Open Source Framework (OSF) (https://osf.io/n4gt9/? view only=5c34285bf73548648361598145de2bc7).

\section{Funding Information}

Parkinson's UK

\section{Supplementary Material}

\section{References}

Babiloni, C., Brancucci, A., Arendt-Nielsen, L., Del Percio, C., Babiloni, F., Pascual-Marqui, R. D., ... Chen, A. C. N. (2004). Cortical sensorimotor interactions during the expectancy of a go/no-go task: effects of painful stimuli. Behavioral Neuroscience, 118, 925- 
Brefel-Courbon, C., Payoux, P., Thalamas, C., Ory, F., Quelven, I., Chollet, F., ... Rascol, O. (2005). Effect of levodopa on pain threshold in Parkinson's disease: A clinical and positron emission tomography study. Movement Disorders, 20, $1557-1563$.

Breivik, H., Collett, B., Ventafridda, V., Cohen, R., \& Gallacher, D. (2006). Survey of chronic pain in Europe: Prevalence, impact on daily life, and treatment. European Journal of Pain, 10, 287-333.

Brown, C. A., El-Deredy, W., \& Jones, A. K. P. (2014). When the brain expects pain: Common neural responses to pain anticipation are related to clinical pain and distress in fibromyalgia and osteoarthritis. European Journal of Neuroscience, 39, 663-672.

Brown, C. A., \& Jones, A. K. (2008). A role for midcingulate cortex in the interruptive effects of pain anticipation on attention. Clinical Neurophysiology, 119, 2370-2379.

Brown, C. A., \& Jones, A. K. P. (2010). Meditation experience predicts less negative appraisal of pain: Electrophysiological evidence for the involvement of anticipatory neural responses. Pain, 150, 428-438.

Brown, C. A., \& Jones, A. K. P. (2012). Psychobiological Correlates of Improved Mental Health in Patients With Musculoskeletal Pain After a Mindfulness-based Pain Management Program.

Brown, C. A., Seymour, B., Boyle, Y., El-Deredy, W., \& Jones, A. K. P. P. (2008). Modulation of pain ratings by expectation and uncertainty: Behavioral characteristics and anticipatory neural correlates. Pain, 135, 240-250.

Brown, C. A., Seymour, B., El-Deredy, W., \& Jones, A. K. P. (2008). Confidence in beliefs about pain predicts expectancy effects on pain perception and anticipatory processing in right anterior insula. Pain, 139, 324-332.

Brunia, C. H., \& van Boxtel, G. J. (2001). Wait and see. International Journal of Psychophysiology, 43, 59-75.

Burgmer, M., Petzke, F., Giesecke, T., Gaubitz, M., Heuft, G., \& Pfleiderer, B. (2011). Cerebral activation and catastrophizing during pain anticipation in patients with fibromyalgia. Psychosomatic Medicine, 73, 751-759.

Chaudhuri, R., \& Schapira, A. H. (2009). Non-motor symptoms of Parkinson's disease: Dopaminergic pathophysiology and treatment. Lancet Neurology, 8, 464-474.

Clark, J. A., Brown, C. A., Jones, A. K. P., \& El-Deredy, W. (2008). Dissociating nociceptive modulation by the duration of pain anticipation from unpredictability in the timing of pain. Clinical Neurophysiology, 119, 2870-2878. 
Delorme, A., \& Makeig, S. (2004). EEGLAB: An open source toolbox for analysis of single-trial EEG dynamics including independent component analysis. Journal of Neuroscience Methods, 134, 9-21.

Dettmers, C., Adler, T., Rzanny, R., van Schayck, R., Gaser, C., Weiss, T., ... Weiller, C. (2001). Increased excitability in the primary motor cortex and supplementary motor area in patients with phantom limb pain after upper limb amputation. Neuroscience Letters, 307, 109-112.

Djaldetti, R., Shifrin, A., Rogowski, Z., Sprecher, E., Melamed, E., \& Yarnitsky, D. (2004). Quantitative measurement of pain sensation in patients with Parkinson disease. Neurology, 62, 2171-2175.

Fairhurst, M., Wiech, K., Dunckley, P., \& Tracey, I. (2007). Anticipatory brainstem activity predicts neural processing of pain in humans. Pain, 128, 101-110.

Forkmann, K., Grashorn, W., Schmidt, K., Undt, O. F., Buhmann, C., \& Bingel, U. (2017). Altered neural responses to heat pain in drug-naive patients with Parkinson disease. Pain, 158, 1408-1416.

Goetz, C. G., Tilley, B. C., Shaftman, S. R., Stebbins, G. T., Fahn, S., Martinez-Martin, P., ... LaPelle, N. (2008). Movement disorder society-sponsored revision of the Unified Parkinson's disease rating scale (MDS-UPDRS): Scale presentation and clinimetric testing results. Movement Disorders, 23, 2129-2170.

González-Roldán, A. M., Bomba, I. C., Diesch, E., Montoya, P., Flor, H., \& Kamping, S. (2016). Controllability and hippocampal activation during pain expectation in fibromyalgia syndrome. Biological Psychology, 121, 39-48.

Hayden, B. Y., Nair, A. C., McCoy, A. N., \& Platt, M. L. (2008). Posterior cingulate cortex mediates outcome-contingent allocation of behavior. Neuron, 60, 19-25.

van Hecke, O., Torrance, N., \& Smith, B. H. (2013). Chronic pain epidemiology and its clinical relevance. British Journal of Anaesthesia, 111, 13-18.

Hubbard, C. S., Khan, S. A., Keaser, M. L., Mathur, V. A., Goyal, M., \& Seminowicz, D. A. (2014). Altered brain structure and function correlate with disease severity and pain catastrophizing in migraine patients. eNeuro, 1, e20.14.

Iannetti, G. D., Hughes, N. P., Lee, M. C., \& Mouraux, A. (2008). Determinants of laser-evoked EEG responses: Pain perception or stimulus saliency? Journal of Neurophysiology, 100, 815-828.

Kabat-Zinn, J. (1982). An outpatient program in behavioral medicine for chronic pain patients based on the practice of mindfulness meditation: Theoretical considerations and preliminary results. General Hospital Psychiatry, 4, 33-47. 
Kabat-Zinn, J., Lipworth, L., \& Burney, R. (1985). The clinical use of mindfulness meditation for the self-regulation of chronic pain. Journal of Behavioral Medicine, 8, 163-190.

Koyama, T., McHaffie, J. G., Laurienti, P. J., \& Coghill, R. C. (2005). The subjective experience of pain: Where expectations become reality. Proceedings of the National Academy of Sciences of the United States of America, 102, 12950-12955.

Lantz, G., Grave de Peralta, R., Spinelli, L., Seeck, M., \& Michel, C. (2003). Epileptic source localization with high density EEG: How many electrodes are needed? Clinical Neurophysiology, 114, 63-69.

Michel, C. M., Lantz, G., Spinelli, L., De Peralta, R. G., Landis, T., \& Seeck, M. (2004). 28-channel Eeg source imaging in epilepsy: Clinical yield and localization precision. Journal of Clinical Neurophysiology, 21, 71-83.

Miron, D., Duncan, G. H., \& Bushnell, M. C. (1989). Effects of attention on the intensity and unpleasantness of thermal pain. Pain, 39, 345-352.

Misra, G., \& Coombes, S. A. (2015). Neuroimaging evidence of motor control and pain processing in the human midcingulate cortex. Cerebral Cortex, 25, 1906-1919.

Morecraft, R. J., \& Van Hoesen, G. W. (1998). Convergence of limbic input to the cingulate motor cortex in the rhesus monkey. Brain Research Bulletin, 45, 209-232.

Morrison, I., Peelen, M. V., \& Downing, P. E. (2007). The sight of others' pain modulates motor processing in human cingulate cortex. Cerebral Cortex, 17, 2214-2222.

Mouraux, A., Diukova, A., Lee, M. C., Wise, R. G., \& Iannetti, G. D. (2011). A multisensory investigation of the functional significance of the pain matrix. NeuroImage, 54, 2237-2249.

Mouraux, A., \& Iannetti, G. D. (2009). Nociceptive laser-evoked brain potentials do not reflect nociceptive-specific neural activity. Journal of Neurophysiology, 101, 3258-3269.

Mulert, C., Jäger, L., Schmitt, R., Bussfeld, P., Pogarell, O., Möller, H.-J., ... Hegerl, U. (2004). Integration of fMRI and simultaneous EEG: Towards a comprehensive understanding of localization and time-course of brain activity in target detection. NeuroImage, 22, 83-94.

Nasreddine, Z. S., Phillips, N. A., Bédirian, V., Charbonneau, S., Whitehead, V., Collin, I., ... Chertkow, H. (2005). The Montreal Cognitive Assessment, MoCA: A brief screening tool for mild cognitive impairment. Journal of the American Geriatrics Society, 53 , 695-699. 
Nees, F., \& Becker, S. (2017). Psychological processes in chronic pain: Influences of reward and fear learning as key mechanisms Behavioral evidence, neural circuits, and maladaptive changes. Neuroscience, 387, 72-84.

Nees, F., Becker, S., Millenet, S., Banaschewski, T., Poustka, L., Bokde, A., ... IMAGEN consortium (2017). Brain substrates of reward processing and the $\mu$-opioid receptor. Pain, 158, 212-219.

Nègre-Pagès, L., Regragui, W., Bouhassira, D., Grandjean, H., \& Rascol, O. (2008). Chronic pain in Parkinson's disease: The crosssectional French DoPaMiP survey. Movement Disorders, 23, 1361-1369.

Ozturk, E. A., Gundogdu, I., Kocer, B., Comoglu, S., \& Cakci, A. (2016). Chronic pain in Parkinson's disease: Frequency, characteristics, independent factors, and relationship with health-related quality of life. Journal of Back and Musculoskeletal Rehabilitation, 30, 101-108.

Pearson, J. M., Hayden, B. Y., Raghavachari, S., \& Platt, M. L. (2009). Neurons in posterior cingulate cortex signal exploratory decisions in a dynamic multioption choice task. Current Biology, 19, 1532-1537.

Pfingsten, M., Leibing, E., Harter, W., Kröner-Herwig, B., Hempel, D., Kronshage, U., \& Hildebrandt, J. (2001). Fear-avoidance behavior and anticipation of pain in patients with chronic low back pain: A randomized controlled study. Pain Medication, 2, 259266.

Reicherts, P., Wiemer, J., Gerdes, A. B. M., Schulz, S. M., Pauli, P., \& Wieser, M. J. (2017). Anxious anticipation and pain: The influence of instructed vs conditioned threat on pain. Social Cognitive and Affective Neuroscience, 12, 544-554.

Rushworth, M. F. S., Walton, M. E., Kennerley, S. W., \& Bannerman, D. M. (2004). Action sets and decisions in the medial frontal cortex. Trends in Cognitive Sciences, 8, 410-417.

Russo, G. S., Backus, D. A., Ye, S., \& Crutcher, M. D. (2002). Neural activity in monkey dorsal and ventral cingulate motor areas: comparison with the supplementary motor area. Journal of Neurophysiology, 88, 2612-2629.

Schestatsky, P., Kumru, H., Valls-Solé, J., Valldeoriola, F., Marti, M. J., Tolosa, E., \& Chaves, M. L. (2007). Neurophysiologic study of central pain in patients with Parkinson disease. Neurology, 69, 2162-2169.

Seeck, M., Lazeyras, F., Michel, C. M., Blanke, O., Gericke, C. A., Ives, J., ... Landis, T. (1998). Non-invasive epileptic focus localization using EEG-triggered functional MRI and electromagnetic tomography. Electroencephalography and Clinical Neurophysiology, 106, 508-512. 
Shackman, A. J., Salomons, T. V., Slagter, H. A., Fox, A. S., Winter, J. J., \& Davidson, R. J. (2011). The integration of negative affect, pain and cognitive control in the cingulate cortex. Nature Reviews Neuroscience, 12, 154-167.

Silverdale, M. A., Kobylecki, C., Kass-Iliyya, L., Martinez-Martin, P., Lawton, M., Cotterill, S., ... Grosset, D. G. (2018). A detailed clinical study of pain in 1957 participants with early/moderate Parkinson's disease. Parkinsonism \& Related Disorders, 56, $27-32$.

Skogar, O., \& Lokk, J. (2016). Pain management in patients with Parkinson's disease: Challenges and solutions. Journal of Multidisciplinary Healthcare, 9, 469-479.

Tan, L. L., Pelzer, P., Heinl, C., Tang, W., Gangadharan, V., Flor, H., ... Kuner, R. (2017). A pathway from midcingulate cortex to posterior insula gates nociceptive hypersensitivity. Nature Neuroscience, 20, 1591-1601.

Tinazzi, M., Recchia, S., Simonetto, S., Defazio, G., Tamburin, S., Moretto, G., ... Valeriani, M. (2009). Hyperalgesia and laser evoked potentials alterations in hemiparkinson: Evidence for an abnormal nociceptive processing. Journal of the Neurological Sciences, 276, 153-158.

Trébuchon-Da Fonseca, A., Giraud, K., Badier, J.-M., Chauvel, P., \& Liégeois-Chauvel, C. (2005). Hemispheric lateralization of voice onset time (VOT) comparison between depth and scalp EEG recordings. NeuroImage, 27, 1-14.

Vitacco, D., Brandeis, D., Pascual-Marqui, R., \& Martin, E. (2002). Correspondence of event-related potential tomography and functional magnetic resonance imaging during language processing. Human Brain Mapping, 17, 4-12.

Vogt, B. A., Berger, G. R., \& Derbyshire, S. W. G. (2003). Structural and functional dichotomy of human midcingulate cortex. European Journal of Neuroscience, 18, 3134-3144.

Vogt, B. A., Finch, D. M., \& Olson, C. R. (1992). Functional heterogeneity in cingulate cortex: The anterior executive and posterior evaluative regions. Cerebral Cortex, 2, 435-443.

Watson, A., El-Deredy, W., Iannetti, G. D., Lloyd, D., Tracey, I., Vogt, B. A., ... Jones, A. K. P. (2009). Placebo conditioning and placebo analgesia modulate a common brain network during pain anticipation and perception. Pain, 145, 24-30.

Yang, Z., Jackson, T., \& Huang, C. (2016). Neural activation during anticipation of near pain-threshold stimulation among the painfearful. Frontiers in Neuroscience, 10, 342. 